\title{
RANS computations of a confined cavitating tip-leakage vortex
}

\author{
Jean Decaix ${ }^{1, *}$, Matthieu Dreyer ${ }^{2}$, Guillaume Balarac ${ }^{3}$, Mohamed Frahat ${ }^{2}$, Cécile \\ Münch $^{1}$
}

\begin{abstract}
Cavitating tip-leakage vortices appear in several hydrodynamic flows such as marine propellers or Kaplan turbines. Cavitating computations are a challenging topic since several keys issues are an ongoing work such as the definition of a universal mass source term.

The present study focuses on the computations of the tip-leakage vortex including the gap between the blade tip and the side wall. Two computations are performed, one without cavitation and a second one with cavitation. In both cases, the results are compared with experimental data. The cavitation influence is investigated by comparing the cavitating and the non-cavitating cases. A particular attention is focused on the vortex core trajectory, the vorticity field and the vortex core identification. It is shown that, compared to the non-cavitating case, cavitation leads to a vortex trajectory closer to the suction side and the side wall, which can be of importance regarding the cavitation erosion. Furthermore, cavitation modified the

\footnotetext{
${ }^{*}$ Corresponding author.

Email address: jean.decaix@hevs.ch (Jean Decaix )

${ }^{1}$ Univ. of Applied Sciences and Arts, Western Switzerland, Route du Rawyl 47, 1950 Sion,

${ }^{2}$ Ecole Polytechnique Fédérale de Lausanne, Laboratory for Hydraulic Machines, 33 Bis Av. De

${ }^{3}$ Univ. Grenoble Alpes, LEGI, F-38000 Grenoble, France, CNRS, LEGI, F-38000 Grenoble,
} Switzerland. Cour, 1007 Lausanne, Switzerland. France.
\end{abstract}


vorticity field in the vortex core region. The main feature is a misalignment between the high vorticity region and the cavitating region, which opens a discussion regarding the definition of the vortex core.

Keywords: Tip-Leakage Vortex, Cavitation, RANS, OpenFOAM

\section{Introduction}

Tip-leakage vortices (TLV) appears in several industrial application such as airfoils, compressor engines, marine propellers and Kaplan turbines. The present study focuses on the confined TLV, which means that the gap between the blade tip and the side wall is taken into account. This flow configuration is observed for instance in Kaplan turbines. The TLV develops from the leading edge of a blade on the suction side due to the pressure difference between the pressure side and the suction side. The TLV is known to promote cavitation, which leads to several drawbacks such as erosion, flow instabilities or noise [1].

The confined TLV has been experimentally studied in the case of air compressors [2-7]. Numerical computations of these experiments have been performed using Reynolds-Averaged Navier Stokes (RANS) [8, 9] or Large Eddy Simulation (LES) $[10,11]$ modelling. The results show that the tip-leakage flow contains more than one vortex. Indeed, in addition to the TLV, one or more tip-separation vortices have been identified. The tip-separation vortices form at the pressure side of the blade and then move to the suction side through the gap. Furthermore, an induced tip vortex with a counter-rotation compared to the TLV is sometimes observed. You [10] proposed the use of the criterion proposed by Joseph [12] to determine the re-

gion where cavitation can occur. Nevertheless, no cavitation computations have been performed. 
Regarding hydraulic flows, Roussopoulos [13] performed cavitation investigations in relation to the development of the TLV in Kaplan-type turbine. The Particle Image Velocimetry (PIV) measurements are made on reduced simplified two-dimensional geometries. It is found that a semi-spherical casing leads to the greatest danger of cavitation erosion. Furthermore, the anti-cavitation lip tested does not prevent the risk of cavitation erosion although the circulation of the vortex is reduced.

Miorini [14] investigated the TLV in a waterjet pump rotor using PIV method. The TLV is depicted as a combination of several vortex filaments wrapping around the vortex core. The filaments never merge. The TLV displacement is depicted using the images of the vortex in both the blade and the side wall. Due to the interaction between the TLV and the side wall, a part of the boundary layer detaches and rolls up in a vortex with a counter-rotation compared to the TLV. This can be identified as the induced vortex described by You [11]. The TLV breakdowns when it approaches the pressure side of the following blade. The turbulent field shows a high value of the turbulent kinetic energy in the vortex core and in the shear layer. On the contrary, the production of turbulent kinetic energy is present only in the shear layer, which means that the turbulent kinetic energy is transported from the shear layer to the vortex core by means of the vortex filaments. Cavitation visualizations are used to support the TLV evolution in the blade passage.

Dreyer [15] measures the velocity field downstream a 2D NACA0009 profile for various tip gaps in absence of cavitation. These measurements reveal the influence of the gap width on the TLV behaviour. Mainly, the tip gap width is directly link to the presence of a wake or jet flow inside the vortex core. Furthermore, the authors propose a new dimensionless coefficient $\tau / \Gamma_{\infty}^{*}$ with $\tau$ the gap width and $\Gamma_{\infty}^{*}$ the normalised circulation for the largest gap width. For $\tau / \Gamma_{\infty}^{*} \approx 0.2$, the TLV circulation reaches a peak and therefore, such a situation has to be avoided in order to reduce 
the risk of cavitation erosion. Flow visualizations using cavitation as a tracer put in evidence the presence of two vortices in the gap region. The TLV that forms on the suction side of the leading edge and the tip-separation vortex that forms in the gap. The tip-separation vortex moves upward and merges with the TLV. The position of the fusion between the two vortices depends on the gap with. Smaller the gap is, more upstream the fusion takes place. The presence of cavitation in each vortex depends on the gap width. Indeed, the cavitation amount in the tip-separation vortex decreases with the increase in the gap width.

Some numerical investigations of the cavitation TLV focus on the spatial inducer. Watanabe [16] carried out a two-dimensional computations based on the vortex method and a cavity growth modelled using the unsteady Bernoulli equation. Despite the simplicity of the model, the cavity growth as well as the trajectory of the vortex core is correctly captured compared to the experimental data at least for rather large gap width $(\tau>5)$. Using a similar approach, Higashi [17] is able to predict the trajectory of the vortex core for three gap widths with a good accuracy. The maximum radius of the cavity is in qualitative agreement but the position where this maximum occurs and the dynamic of the radius decrease are not well predicted. A three-dimensional inducer has been computed by Okita [18]. Turbulence is modelled using the Detached Eddy Simulation (DES) model [19] and cavitation is modelled using the model proposed by Chen [20]. It is shown that cavitation enhanced the tip-clearance flow. Moreover, the rotation of the backflow cavitation rotates with a speed lower than the rotation speed of the inducer. Nevertheless, such results are not compared with experimental data, which limits the validation of the computation. Recently, the cavitating tip leakage vortex has been investigated in an axial flow pump [21, 22]. The modelling is based on a homogeneous model coupled with the SST $k-\omega$ model including the Reboud correction for the eddy viscosity [23]. The 
simulations show that the tip leakage vortex influences the suction side perpendicular cavitating vortex formation.

Non-confined cavitating TLV are also computed in the case of marine propellers. Hsiao [24] investigated the cavitation inception inside the tip vortex by solving the RANS equations coupled with the Baldwin-Lomax algebraic turbulence model. Cavitation is modelled using either a spherical bubble model based on the RayleighPlesset equation or a non-spherical bubble model [25]. The results show that the definition of the cavitation inception and the non-spherical deformations can have an important role in order to predict the cavitation inception. A use of a surfaceaveraged pressure in the spherical model significantly improves the results and bring them closer to the non-spherical model. Bensow [26] performs Implicit Large Eddy Simulation (I-LES) computations of a marine propeller using the cavitation model proposed by Kunz [27]. The comparison of flow pictures with the iso-surface of the volume fraction provided by the computation shows a qualitative agreement even if cavitation in the TLV is under-resolved.

In the present paper, the TLV that develops at the tip of a NACA0009 profile mounted in a rectangular channel is investigated for one gap width. Previous noncavitation computations for two gap widths have been carried out using RANS and LES turbulence models [28]. The comparison of the results with experimental data [15] and between the RANS and LES computations show that the RANS computations are able to capture the mean flow with accuracy. Therefore, RANS modelling is used to perform the cavitating computation. First, the computations without and with cavitation are compared with the experimental data. Then, the influence of cavitation on the TLV is discussed by comparisons with the non-cavitating case. Finally, a special focus on the cavitating TLV core is carried out. 


\section{Numerical tools}

The computations are carried out using the OpenFOAM software release 2.1.0. This software is based on an orientated object framework [29, 30].

For the non-cavitating case, the unsteady incompressible RANS equations using the Boussinesq assumptions for the turbulent stresses are solved:

$$
\begin{gathered}
\nabla \cdot \vec{u}=0 \\
\frac{\partial \vec{u}}{\partial t}+\nabla \cdot(\vec{u} \otimes \vec{u})-\nabla \cdot\left(\nu_{e f f} \nabla \vec{u}\right)=-\nabla\left(\frac{p}{\rho}\right)
\end{gathered}
$$

with the effective viscosity $\nu_{\text {eff }}=\nu+\nu_{t} . \quad \nu$ is the kinematic viscosity and $\nu_{t}$ is the turbulent viscosity. The turbulent viscosity $\nu_{t}$ is computed using the SST $k-\omega$ model [31]. The log wall law is assumed to compute the shear stress in the first cell layer. The time derivatives is computed with the backward second order scheme. The convective flux is discretized with the Total Variation Diminishing (TVD) scheme named "limitedLinear" specific to OpenFOAM. The limiter is de-

fined as $\Psi\left(r_{f}\right)=\max (\min (2 r, 1), 0)$. The time derivative is discretized using the second order backward scheme. The set of equations is solved by using a coupled SIMPLE/PISO algorithm.

For the cavitating case, the homogeneous mixture approach is used. Therefore, the two phases share the same velocity, pressure and temperature. Furthermore, in the present work, the temperature is assumed constant. The mixture density $\rho$ and the mixture kinematic viscosity $\nu$ are computed as the phase average of the phase value:

$$
\begin{aligned}
& \rho=\alpha_{L} \rho_{L}+\left(1-\alpha_{L}\right) \rho_{V} \\
& \nu=\alpha_{L} \nu_{L}+\left(1-\alpha_{L}\right) \nu_{V}
\end{aligned}
$$

with: 
- $\rho_{L}=1000 \mathrm{~kg} \mathrm{~m}^{-3}$, the liquid density.

- $\nu_{L}=9 \cdot 10^{-7} \mathrm{~m}^{2} \mathrm{~s}^{-1}$, the liquid viscosity.

- $\rho_{V}=0.02 \mathrm{~kg} \mathrm{~m}^{3}$, the vapour density.

- $\nu_{V}=4.473 \cdot 10^{-4} \mathrm{~m}^{2} \mathrm{~s}^{-1}$, the vapour viscosity.

- $\alpha_{L}$, the liquid volume fraction.

The set of equations to be solved is:

$$
\begin{aligned}
\frac{\partial \alpha_{L}}{\partial t}+(\vec{u} \cdot \nabla) \alpha_{L} & =S \\
\nabla \cdot \vec{u} & =\left(\frac{\rho_{V}-\rho_{L}}{\rho}\right) S \\
\frac{\partial \rho \vec{u}}{\partial t}+\nabla \cdot(\rho \vec{u} \otimes \vec{u})-\nabla \cdot\left(\mu_{e f f} \nabla \vec{u}\right) & =-\nabla p
\end{aligned}
$$

Equation 5 is the transport equation for the liquid volume fraction with $S$ the source term. It is modelled using a slightly modified version of the model proposed by Kunz [27] (effects of non-condensable gases are non-considered), which splits the source term ${ }^{4}$ in a condensation term $m_{c}$ and a vaporisation term $m_{v}$ :

$$
\begin{aligned}
S & =m_{c}+m_{v} \\
m_{c} & =\frac{\rho}{\rho_{L} \rho_{V}} C_{c} \frac{\rho_{V}}{t_{\infty}} \alpha_{\text {Llim }}^{2} \frac{\max \left(p-p_{\text {sat }} ; 0\right)}{\max \left(p-p_{\text {sat }} ; 0.01 p_{\text {sat }}\right)} \\
m_{v} & =\frac{\rho}{\rho_{L} \rho_{V}} C_{v} \frac{\rho_{V}}{\frac{1}{2} \rho_{L} U_{\infty}^{2} t_{\infty}} \min \left(p-p_{\text {sat }} ; p_{0}\right)
\end{aligned}
$$

The constant $p_{0}$ is set to 0 and $\alpha_{\text {Llim }}$ is defined as $\min \left(\max \left(\alpha_{L}, 0\right), 1\right)$, which preserve the boundedness of $\alpha_{L}$ during the iterative process. After calibration, the constants

\footnotetext{
${ }^{4}$ In the OpenFOAM formulation, the source terms are multiplied by the factor $\rho /\left(\rho_{L} \rho_{V}\right)$ compared to the formulation proposed by Kunz
} 
$C_{v}$ and $C_{c}$ are set to $C_{v}=3500$ and $C_{c}=500$. the others parameters are set as follow: $U_{\infty}=u_{\text {inlet }}=10.2 \mathrm{~m} \mathrm{~s}^{-1}, t_{\infty}=L_{r e f} / U_{\infty}=0.005 \mathrm{~s}$ and $p_{\text {sat }}=2300 \mathrm{~Pa}$. The reference length scale $L_{r e f}$ is set to $L_{r e f}=c / 2$ with $c$ the chord length.

The set of equations is solved using a coupled SIMPLE/PISO algorithm. The transport equation for the liquid volume fraction is solved using a Multidimensional Universal Limiter for Explicit Solution (MULES) specific to OpenFOAM [32, 33]. Such a method introduces a counter gradient to limit the diffusion of the interface and required to be coupled with a high order scheme for the discretization of the convective term. The transport equation for the liquid volume fraction $\alpha_{L}$ (equation 5) is solved in a conservative form, which requires to treat the velocity divergence as an explicit source term. The convective flux is discretized using the Van Leer limiter [34]. For the momentum and turbulence equations, the convective flux is discretized using the "limitedLinear" scheme. The eddy viscosity is computed using the SST $k-\omega$ model [31]. It is assumed that wall laws apply in two-phase flows. This assumption is supported by recent results [35]. Identically to the single test case, the time derivative is discretized using the second order backward scheme.

\section{Test case}

The hydrofoil is made of a NACA0009 profile with an originally chord of $c_{0}=$ $0.11[\mathrm{~m}]$ and a span of $0.15[\mathrm{~m}][36]$. The shape of the blade is computed using equations (11) and (12).

$$
\begin{aligned}
& \frac{y_{b}}{c_{0}}=a_{0}\left(\frac{x}{c_{0}}\right)^{1 / 2}+a_{1}\left(\frac{x}{c_{0}}\right)+a_{2}\left(\frac{x}{c_{0}}\right)^{2}+a_{3}\left(\frac{x}{c_{0}}\right)^{3} \quad \text { for } \quad 0 \leq \frac{x}{c_{0}} \leq 0.5 \\
& \frac{y_{b}}{c_{0}}=b_{0}+b_{1}\left(1-\frac{x}{c_{0}}\right)+b_{2}\left(1-\frac{x}{c_{0}}\right)^{2}+b_{3}\left(1-\frac{x}{c_{0}}\right)^{3} \quad \text { for } \quad 0.5 \leq \frac{x}{c_{0}} \leq 1
\end{aligned}
$$


with:

$$
\begin{array}{llll}
a_{0}=0.1737 & a_{1}=-0.2422 & a_{2}=0.3046 & a_{3}=-0.2657 \\
b_{0}=0.0004 & b_{1}=0.1737 & b_{2}=-0.1898 & b_{3}=0.0387
\end{array}
$$

In the present case, the blade is truncated, therefore the chord is reduced to $c=0.1$ m. The origin of the axis corresponds to the centre of rotation of the blade pitch. The hydrofoil is mounted in the channel of the EPFL high-speed cavitation tunnel, which is a close loop with a test section measuring $0.15 \times 0.15 \times 0.750 \mathrm{~m}^{3}$ [37]. The incidence of the profile is set to $i=10^{\circ}$ and the gap $\tau$ is set to $\tau / c=0.1$.

Numerical computations are carried out on a domain that extends 2 chords upstream the leading edge and 5 chords downstream the trailing edge (see figure 1). The reference structured mesh contains around 2 millions of nodes (see figure 2). The gap width is filled with 30 nodes in the spanwise direction (see the Appendix for the simulation and mesh convergence assessment).

The inlet velocity is set to a uniform value $u_{\text {inlet }}=10.2 \mathrm{~m} \mathrm{~s}^{-1}$, which corresponds to a Reynolds number based on the blade chord $R e_{c}=10^{6}$. At the outlet, a gradient free condition is set for all the variables. The pressure reference is set in an upstream section $(x / c=-1.3)$ in agreement with the experimental measurement section. The pressure value will be discussed in the result part. The cavitation number $\sigma_{u}$ is measured in the upstream section located at $x / c=-1.3$ where pressure measurements are performed. It is computed as:

$$
\sigma_{u}=\frac{p_{\text {ref }}-p_{\text {sat }}}{\frac{1}{2} \rho u_{\text {inlet }}^{2}}
$$

with $p_{\text {ref }}$ the pressure reference in the cross section located at $x / c=-1.3, p_{\text {sat }}$ the saturated vapour pressure set to $2300 \mathrm{~Pa}$ and $\rho$ the liquid density equal to $1000 \mathrm{~kg} \mathrm{~m}^{-3}$. For the cavitating case, the cavitation number is $\sigma_{u}=2.01$. 
The unsteady computations are run with a time step set to $\Delta t=10^{-5} \mathrm{~s}$. They are started form a steady computations and the time-averaged quantities are computed over $T=0.1 \mathrm{~s}$ after the flow is developed. At each time step, two loops are set both for the PISO loop and the SIMPLE loop. A multigrid solver is used to solve the pressure equation whereas, a preconditioned bi-conjugated gradient solver is used for the momentum and turbulence conservation equations. The maximum CFL number is around 2.6 whereas the mean CFL is lower than 0.05. One time step requires approximately $60 \mathrm{~s}$ to be achieved using 8 cores of an Intel ${ }^{\circledR}$ Xeon ${ }^{\circledR}$ X5650 (2.67 $\mathrm{GHz}) \mathrm{CPU}$.

For the non-cavitating case, the 3D velocity field is available at three downstream sections located at $x / c=1, x / c=1.2$ and $x / c=1.5$ (see figure 3). The velocity measurements are performed using stereo-PIV. For the cavitating case, only instantaneous side views of the cavitating TLV are available.

\section{Results}

The results are displayed using a dimensionless representation based on the reference velocity $u_{\text {ref }}=u_{\text {inlet }}=10.2 \mathrm{~m} \mathrm{~s}^{-1}$ and the reference length scale $l_{\text {ref }}=c=0.1$ $\mathrm{m}$. For instance, the dimensionless velocity is given by $u^{*}=u / u_{\text {ref }}$.

\subsection{Non-cavitating TLV}

The global feature of the flow is shown using the Q-criterion computed using the time-averaged velocity field (see figure 4). The tip-leakage vortex and the tipseparation vortex are clearly identified. The two vortices merge in a single vortex that evolves downstream. This topology is in agreement with the experimental visualizations using cavitation as a passive tracer [15] and previous LES computations [28]. 
The time-averaged dimensionless velocity components at the first downstream section $x / c=1$ is compared between the experiment and the computation (see figure 5). Regarding the axial component $u^{*}$, the accelerated region close to the vortex core is accurately located with nevertheless an underestimation of the velocity magnitude. Focusing on the transverse components $v^{*}$ and $w^{*}$, the magnitude of the components is predicted in agreement with the measurements. However, it is noticeable that the gradient of these components at the vortex core is diffused. A part of the explanation is the size of the mesh that is too coarse compared to the size of the vortex core. Furthermore, the numerical and model diffusions play also a role. Previous LES computations on a finer mesh (200 millions of elements) show an improvement of the gradient resolution even if it is still slightly diffused compared to the experiment [28]. Such a result supports the present choice to not refine the mesh in the vortex core.

The time-averaged dimensionless axial vorticity component $\omega_{x}^{*}$ at the first measurement section $x / c=1$ is displayed on figure 6 . As explained above, the diffusion of the transverse gradients lead to an underestimation of the axial vorticity in the vortex core region. In order to determine the region where the vorticity is under-estimated, the computational results are interpolated on the experiment PIV grid measurement. Then, the ratio between the experimental and numerical dimensionless axial vorticity $\omega_{x_{E x p e}}^{*} / \omega_{x_{R A N S}}^{*}$ is computed and plotted on figure 7 . The magnitude of the axial vorticity is underestimated mainly at the vortex core, whereas it is captured outside the vortex core. The limit of the vortex is shown by the arc of circle with the low and high ratio values.

Tables 1 and 2 give the pitchwise $y / c$ and spanwise $z / c$ position of the vortex core at the three measurement sections. The vortex core position is detected using the 
maximum of the axial vorticity $\omega_{x}$. The absolute error $\delta$ is computed as :

$$
\delta=\frac{\left|s_{\text {RANS }}-s_{\text {Expe }}\right|}{s_{\text {Expe }}} \times 100
$$

with $s$ the pitchwise or spanwise position of the vortex core. The computational results are in agreement with the experimental data since the absolute error is close or lower than $10 \%$ except for the pitchwise position at $x / c=1.5$. The $10 \%$ error corresponds to approximately one millimetre, which is the order of magnitude of the cell size.

\subsection{Cavitating TLV}

Since the vorticity in the core of the TLV is under-resolved by the RANS computation, the pressure drop in the core cannot be predicted accurately compared to the experiment. Therefore, if the computation is performed at the same cavitation number $\sigma_{u}=2.01$ than the experiment, no cavitation occurs. In order to produce cavitation in the TLV, the computation is carried out at a lower cavitation number $\sigma_{u}=1.3$. This value corresponds to the one that provides the more developed cavitating tip vortex. For lower values, the computation becomes unstable whereas for a higher value, the cavitating tip vortex is less extended.

For this flow configuration, the tip-leakage vortex is displayed on figure 8 using an iso-surface of the non-dimensional Q-criterion $\left(Q^{*}=1.5\right)$ and an iso-surface of the time-averaged liquid volume fraction $\alpha_{\text {Lmean }}=0.9$. As for the non-cavitating case, the tip-leakage vortex and the tip-separation vortex merge in one single vortex. The fusion of the two vortices is delayed downstream in the cavitating case. Cavitation occurs inside the tip-leakage vortex and in the shears layer along the blade tip. No cavitation is observed in the tip-separation vortex when it moves from the gap to the tip-leakage vortex. 
The comparison with the experiment is shown on figure 9. The position of the cavitating TLV is well captured above the blade even if the size of the cavitation core is slightly larger in the computation than in the experiment. The cavitation in the shear layer provided by the computation stops at roughly the same place than the cavitation clouds observed on the experimental picture.

Since no quantitative experimental data are available for the cavitating case, the analysis of the cavitating flow is performed using only numerical results.

Figure 10 compares the time-averaged streamwise velocity component between the non-cavitating and the cavitating cases. Without cavitation, the flow is attached on the suction side, whereas it detaches in presence of cavitation. This feature is illustrated more in detail in figure 11, which displays an iso-surface of the dimensionless Q-criterion and the contours of the dimensionless streamwise velocity component in three cross planes for both cavitating and non-cavitating cases. The larger wake observed for the cavitating case seems to influence the trajectory of the vortex core downstream the trailing edge as shown on figure 12. The vortex core position is determined using the maximum of the Q-criterion. The wake confines the vortex close to the side wall (located at $z / c=0$ ) and prevents the vortex to move upward. This feature can be explained by the competition between the image of the vortex in the side wall that leads to the upward moving and the suction effect due to the low pressure in the wake that provokes downward moving. Since cavitation in the vortex stops close to the trailing edge, an influence of cavitation on the vortex trajectory more downstream the trailing edge cannot be excluded. Above the blade, the position of the tip-leakage vortex core (figure 13) is not strongly influenced by the presence of cavitation. In the spanwise direction, the difference observed from $x / c=0.1$ to $x / c=0.4$ is likely due to the fusion between the TLV and the tipseparation vortex that takes place slightly downstream in the cavitating case. 
Figure 14 displays the time-averaged liquid volume fraction and an iso-contour of the Q-criterion in five transversal planes along the blade. At the planes located at $x / c=-0.3$ and $x / c=-0.1$, the minimum of the liquid volume fraction is encircled by the iso-contour of the Q-criterion, which means that cavitation occurs at the vortex core. However, at the three downstream planes, the minimum value of the liquid volume fraction is not located inside the iso-contour of the Q-criterion. The misalignment between the minimum of the liquid volume fraction and the maximum of the Q-criterion is related to the fusion of the tip-leakage vortex and the tip-separation vortices originating from the gap. Therefore, in this region the criteria used to determine the vortex center is not straightforward and a specific investigation is required.

\subsection{Cavitation and vortex core identification}

In order to better understand the topology of the TLV core, a detailed investigation have been carried out.

First, the location of the TLV core has been determined using several criteria:

- Simple criteria [38]:

$\diamond$ Minimum of pressure.

$\diamond$ Maximum of axial vorticity.

$\diamond$ Minimum of liquid volume fraction.

- Mathematical criteria derived for incompressible flows [39]:

$\diamond$ Maximum of the Q-criterion that works also for compressible flows [40].

$\diamond$ Maximum of the $-\lambda_{2}$ criterion.

Figure 15 displays the pitchwise and the spanwise position above the blade of the TLV core identified using each of the criteria aforementioned for the cavitating and 
the non-cavitating cases. Focusing on the non-cavitating case, the Q-criterion and the $\lambda_{2}$ criterion give the same pitchwise and spanwise position. The maximum of the axial vorticity is at the most of the positions in agreement with these two criteria. The only difference observed is located at $x / c=0.2$ that corresponds to the place where the TLV merges with the tip-separation vortex. In the spanwise direction, the pressure minimum gives a position of the TLV in accordance with the other criteria (at $x / c=-0.4$, the pressure minimum is not present because it is located in the boundary layer originating from the leading edge and not in the vortex region), whereas in the pitchwise direction, the vortex core is often positioned slightly lower. Looking at the cavitation case, between $x / c=-0.4$ and $x / c=0$, all the criteria predict the same pitchwise position, whereas differences are observed in the spanwise position even between the Q-criterion, the $\lambda_{2}$ criterion and the maximum of the axial vorticity. At $x / c=-0.4$ and $x / c=-0.3$, the spanwise difference between the minimum of the liquid volume fraction and the others criteria can be explained by the development of the cavitation at the leading edge. Downstream the fusion between the TLV and the tip-separation vortex that takes place approximately at $x / c=0.1$, the $\mathrm{Q}$-criterion, the $\lambda_{2}$ criterion and the maximum of the axial vorticity give the same pitchwise and spanwise position of the TLV core. The minimum of the liquid volume fraction shows a lower pitchwise and spanwise position. The pressure minimum is located between the minimum of the liquid volume fraction and the Q-criterion.

The previous analysis suggests that the presence of cavitation leads to a misalignment of the vortex core position depending on the criteria used. The criteria based on the velocity field do not give the same results than the ones based on the pressure field. 
To better understand this behaviour, the following vorticity equation

$$
\frac{\partial \vec{\omega}}{\partial t}+(\vec{u} \cdot \nabla) \vec{\omega}=(\vec{\omega} \cdot \nabla) \vec{u}-\vec{\omega} \nabla \cdot \vec{u}+\nu \nabla^{2} \vec{\omega}+\nu_{t} \nabla^{2} \vec{\omega}+\frac{1}{\rho^{2}} \nabla \rho \wedge \nabla p
$$

is investigated term by term. With:

- $(\vec{\omega} . \nabla) \vec{u}$, the term responsible for the vortex stretching.

- $\vec{\omega} \nabla \cdot \vec{u}$, the term due to the flow compressibility.

- $\nu \nabla^{2} \vec{\omega}$, the viscous term.

- $\nu_{t} \nabla^{2} \vec{\omega}$, the turbulent term.

- $\frac{1}{\rho^{2}} \nabla \rho \wedge \nabla p$, the baroclinic torque.

It is obvious that the compressible term and the baroclinic torque are equal to zero for the non-cavitating case. In presence of cavitation, these terms are not any more null since the divergence of the velocity is expressed by equation (6) and the pressure and the density are not linked by a barotropic equation of state.

The terms that compose the vorticity equation are analyzed in the cross section located at $x / c=0$. They are expressed in a dimensionless form by dividing all the terms of equation (15) by the quantity $u_{r e f}^{2} / l_{r e f}^{2}=10^{4} \mathrm{~s}^{-2}$, which is the dimension of the term $\partial \vec{\omega} / \partial t$.

The dimensionless streamwise vortex stretching, viscous and turbulent terms are represented on figure 16 both for the non-cavitating and cavitating cases. In addition, an iso-contour of the Q-criterion and of the liquid volume fraction is also plotted. Looking at the vortex stretching term, it is rather negative for the cavitating case inside the vortex core and seems to be null at the vortex core for the non-cavitating case. The viscous term is negligible in the non-cavitating case compared to the 
cavitating computation due to the larger value of the vapour kinematic viscosity $\nu_{V}=4 \cdot 10^{-4}$ compared to the liquid kinematic viscosity $\nu_{L}=9 \cdot 10^{-7}$. The turbulent term is negative inside the non-cavitating vortex, whereas it shows rather a positive value inside the cavitating vortex. It is noticeable that for the cavitating computation, the turbulent term is also negative inside the iso-contour of the Q-criterion. Figure 17 shows the non-dimensional streamwise component of the baroclinic torque and the compressible term that are specific to the cavitating computation. Inside the cavitating vortex, the two terms are positive. However, the compressible term acts as a negative source term due to the minus sign in the vorticity equation (see equation 15). The non-dimensional streamwise component of the right-hand side (RHS) term of the vorticity equation is then displayed on figure 18 for the both cases. The RHS term is largely negative for the cavitating case compared to the non-cavitating case mainly due to the compressible and the viscous terms. Furthermore, it is negative both inside the vortex core delimited by the Q-criterion and the vortex core delimited by the liquid volume fraction. Therefore, the vorticity inside the vortex core decreases in the cavitating case, whereas it is rather transported in the non-cavitating case without damping. Such a difference could explain the difference between the criteria used to identify the vortex core and finally the presence of two vortex cores in the cavitating case: one identified as the the high vorticity core and the second corresponding to the cavitating core.

Since vorticity is diffused by the cavitating core, the replacement of the vorticity in the vicinity of the cavitating TLV seems to be due to the fusion process between the TLV and the tip-separation vortices originating from the gap region. This observation can be related with the distribution of the turbulent kinetic energy in the cavitating TLV studied by Miorini [14]. Miorini observed that the production of turbulent kinetic energy is null in the vortex core whereas it is maximal in the shear layer 
originating from the gap. He concludes that the turbulent kinetic energy observed in the TLV core is transported from the shear layer to the vortex core by vortex filaments. Figure 19 representing the turbulent kinetic energy and the turbulent kinetic production term supports this mechanism. The shear layer is the place where turbulent kinetic energy and vorticity are produced. Then the tip-separation vortex transports the turbulent kinetic energy and the vorticity inside the TLV, where the vorticity is diffused by cavitation due to compressible and viscous effects.

The conclusions drawn in this section have to be confirmed by computations performed with improved two-phase flow modelling that for instance do not considered the homogeneous assumptions and that take into account a possible turbulent/cavitation interaction.

\section{Conclusion}

The development of the TLV including the gap between the blade tip and the side-wall has been numerically investigated with and without cavitation. The gap width was set to $10 \%$ of the chord length and the incidence was set to $10^{\circ}$.

Without cavitation, the RANS computation shows a good agreement with the experimental data available downstream the blade. The topology of the flow put in evidence the presence of the tip-leakage vortex and the tip-separation vortex. According to the experimental visualizations using cavitation as a passive tracer, the two vortices merge in a single one at the blade mid-chord. Furthermore, the position of the vortex core and the velocity field are well captured compared to the experiment. However, the vortex core is under-resolved as shown by the under-estimation of the axial vorticity at the vortex core. The under-estimation is linked to the diffusion of the transverse velocity gradients at the vortex core. The diffusion is a result of the mesh and numerical diffusion. As a consequence, the pressure drop in the 
vortex core is also underestimated.

Therefore, the cavitating RANS computation is performed at a cavitation number $\sigma_{u}=1.3$ lower than the experimental one $\sigma_{u}=2.1$. The comparison with the experiment regarding the shape of the cavitating TLV shows a good agreement except that cavitation stops further upstream in the simulation. Nevertheless, the cavitating and the non-cavitating computations are compared. The cavitating computation reveals the presence of a thick cavitation layer at the leading edge, which causes the detachment of the boundary layer on the suction side of the blade. Therefore, compared to the single-phase computation, the pressure field on the suction side is modified and a larger wake is observed downstream the blade. These differences influence the vortex core trajectory since the cavitating vortex evolves closer to the suction side and the side wall. Regarding the cavitation erosion risk, such a result has to be taken into account even if the effect of a rotating motion is not considered in the present simulation.

A deeper investigation of the cavitating TLV has been performed. It is revealed that upstream the fusion between the TLV and the tip-separation vortex, cavitation develops inside the vortex core. On the contrary, downstream the fusion, cavitation zones and high vorticity zones do not match between them. To better understand this difference, a comparison of the criteria used to identify a vortex core has been done. Without cavitation, the Q-criterion, the $\lambda_{2}$ criterion and the maximum of axial vorticity predict the same position of the vortex core. The minimum of pressure is in agreement with these criteria regarding the spanwise position of the vortex whereas the pitchwise position is slightly lower. For the cavitating case, it is shown that the cavitating region does not match with the vortex core determined with the usual criteria on a large part of the TLV trajectory. To explain the difference between the cavitating and the non-cavitating case, the terms of the axial vorticity equation 
are investigated in a cross plane located at mid-chord. Without cavitation, the RHS of the vorticity equation is close to zero, which means that the axial vorticity is transported by the flow without damping effect. On the contrary, for the cavitating case, the RHS of the vorticity equation is strongly negative, which means that the axial vorticity decreases in the vortex core. Consequently, two vortex cores can be considered: one corresponding to the region of highest vorticity and a second identified as the region of highest vapour volume fraction. Since the vorticity decrease in the cavitating TLV, a mechanism is proposed to explain the presence of high vorticity regions. Vorticity is produced in the shear layer located in the gap and then transported to the TLV by the tip-separation vortex. Finally, due to compressibility and viscous effects inside the cavitating TLV core, the vorticity is diffused. This mechanism is also supported for the turbulent kinetic energy and is in agreement with the experimental work carried out by Miorini [14].

The present study suggests that a full resolved computation of a vortex core is still a challenging task. Improved two-phase flow models, numerical schemes and turbulence are certainly required in order to accurately capture the vortex core in detail. Nevertheless, by decreasing the pressure level in the computational domain, the cavitating TLV has been captured with a qualitative agreement compared to the experimental data. It is also suggested that cavitation could influence the TLV vortex

particularly regarding the vortex trajectory, the vorticity field and the definition of the vortex core. These conclusions have to be supported in the future by additional experimental and numerical studies.

\section{Acknowledgements}

The authors are very grateful to the Competence Center in Energy and Mobility (CCEM), Swisselectric Research and the foundation The Ark through the program 
The Ark Energy for their financial support. 
[1] R. E. A. Arndt, Cavitation in Vortical Flows, Annual Review of Fluid Mechanics 34 (2002) 143-175.

[2] S. Kang, C. Hirsch, Experimental Study on the Three-Dimesnional Flow Within a Compressor Cascade With Tip Clearance: Part I - Velocity and Pressure Fields., Journal of Turbomachinery 115 (1993) 435-467.

[3] S. Kang, C. Hirsch, Experimental Study on the Three-Dimensional Flow Within a Compressor Cascade With Tip Clearance: Part II - The Tip Leakage Vortex, Journal of Turbomachinery 115 (1993) 444-452.

[4] B. Lakshminarayana, M. Pouagare, R. Davino, Three-dimensional flow-field in the tip region of a compressor rotor passage, part 1 : mean velocity profiles and annulus wall boundary layer, Journal of Engineering for gas turbines and power 104 (1982) 760-771.

[5] B. Lakshminarayana, M. Pouagare, R. Davino, Three-dimensional flow-field in the tip region of a compressor rotor passage, part 2 : turbulence properties, Journal of Engineering for gas turbines and power 104 (1982) 772-781.

[6] C. Muthanna, W. J. Devenport, Wake of a Compressor Cascade with Tip Gap, Part 1 : Mean Flow and Turbulence Structure, AIAA Journal 42 (2004) 23202331.

[7] Y. Wang, W. J. Devenport, Wake of a Compressor Cascade with Tip Gap, Part 2 : Effects of Endwall Motion, AIAA Journal 42 (2004) 2332-2340.

[8] S. Kang, C. Hirsch, Numerical simulation of three-dimensional viscous flow in a linear compressor cascade withtip clearance, Journal of Turbomachinery 118 (1996) 492-505. 
[9] D. Borello, K. Hanjalic, F. Rispoli, Computation of tip-leakage flow in a linear compressor cascade with a second-moment turbulence closure, International Journal of Heat and Fluid Flow 28 (2007) 587-601.

[10] D. You, M. Wang, P. Moin, R. Mittal, Effects of tip-gap size on the tip-leakage flow in a turbomachinery cascade, Physics of Fluids 18 (2006).

[11] D. You, M. Wang, P. Moin, R. Mittal, Large-eddy simulation analysis of mechanisms for viscous losses in a turbomachinery tip-clearance flow, Journal of Fluid Mechanics 586 (2007) 177-204.

[12] D. D. Joseph, Cavitation and the state of stress in a flowing liquid, Journal of Fluid Mechanics 366 (1998) 367-378.

[13] K. Roussopoulos, P. A. Monkewitz, Measurements of Tip Vortex Characteristics and the Effect of an Anti-Cavitation Lip on a Model Kaplan Turbine Blade, Flow, Turbulence and Combustion 64 (2000) 119-144.

[14] R. L. Miorini, J. Katz, The Internal Structure of the Tip Leakage Vortex Within the Rotor of an Axial Waterjet Pump, Journal of Turbomachinery 134 (2012).

[15] M. Dreyer, J. Decaix, C. Münch, M. Farhat, Mind the gap: a new insight on the tip leakage vortex using stereo-PIV, Experiment in Fluids 55 (2014).

[16] S. Watanabe, H. Seki, S. Higashi, K. Yokota, Y. Tsujimoto, Modeling of 2D Leakage Jet Cavitation as a Basic Study of Tip Leakage Vortex Cavitation, Journal of Fluids Engineering 123 (2001) 50-56.

[17] S. Higashi, Y. Yoshida, Y. Tsujimoto, Tip leakage vortex cavitation from the tip clearance of a single hydrofoil, JSME International Journal 45 (2002). 
[18] K. Okita, K. Ugajin, H. Matsumoto, Numerical Analysis of the Inlfuence of the Tip Clearence Flows on the unsteady cavitating flows in a three-dimensional Inducer, Journal of Hydrodynamics 21 (2009) 34-40.

[19] P. R. Spalart, W. H. Jou, M. Strelets, S. R. Allmaras, Comments on the feasibility of LES for wings and on a hybrid RANS/LES approach, in: Advances in DNS/LES, volume 1, 1997, pp. 4-8.

[20] Y. Chen, S. D. Heister, Two-phase modeling of cavitated flows, Computers \& Fluids 24 (1995) 799-809.

[21] D. Zhang, W. Shi, D. Pan, M. Dubuisson, Numerical and Experimental Investigation of Tip Leakage Vortex Cavitation Patterns and Mechanisms in an Axial Flow Pump, Journal of Fluids Engineering 137 (2015).

[22] D. Zhang, L. Shi, W. Shi, R. Zhao, H. Wang, B. P. M. B. V. Esch, International Journal of Multiphase Flow Numerical analysis of unsteady tip leakage vortex cavitation cloud and unstable suction-side-perpendicular cavitating vortices in an axial flow pump, International Journal of Multiphase Flow 77 (2015) 244259. doi:10.1016/j.ijmultiphaseflow.2015.09.006.

[23] J.-L. Reboud, B. Stutz, O. Coutier, Two-phase flow structure of cavitation: experiment and modelling of unsteady effects, in: $3^{\text {rd }}$ International Symposium on Cavitation CAV1998, Grenoble, France, 1998.

[24] C.-T. Hsiao, G. Chahine, Prediction of tip vortex cavitation inception using coupled spherical and nonspherical bubble models and Navier-Stokes computations, Journal of Marine Science and Technology 8 (2004) 99-108. 
[25] C.-T. Hsiao, G. L. Chahine, Numerical Simulation of Bubble Dynamics in a Vortex Flow Using Navier- Stokes Computations and Moving Chimera Grid Scheme, Chimera (2001) 1-10.

[26] R. Bensow, G. Bark, Implicit LES predictions of the cavitating flow on a propeller, Journal of Fluids Engineering 132 (2010).

[27] R. Kunz, D. Boger, D. Stinebring, T. Chyczewski, J. Lindau, H. Gibeling, S. Venkateswaran, T. Govindan, A preconditioned Navier-Stokes method for two-phase flows with application to cavitation prediction, Computers \& Fluids 29 (2000) 849-875.

[28] J. Decaix, G. Balarac, M. Dreyer, M. Farhat, C. Münch, RANS and LES computations of the tip-leakage vortex for different gap widths, Journal of Turbulence 16 (2015) 309-341.

[29] H. Weller, G. Tabor, H. Jasak, C. Fureby, A tensorial approach to computational continuum mechanics using objected-oriented techniques, Computers in Physics 12 (1998) 620-631.

[30] H. Jasak, Error analysis and estimation for the finite volume method with applications to fluid flows, Ph.D. thesis, University of London, Imperial College, 1998.

[31] F. Menter, Improved Two-Equation $k-\omega$ Turbulence Models for Aerodynamic Flows, Technical Report, NASA Technical Memorandum 103975, 1992.

[32] K. Kissling, J. Springer, Jasak, Hrvoje, S. Schütz, K. Urban, M. Piesche, A coupled pressure based solution algorithm based on the volume-of-fluid approach 
for two or more immiscible fluids, in: V European Conference on Computational Fluid Dynamics ECCOMAS CFD2010, 2010.

[33] S. M. Damián, An extended Mixture Model for the Simultaneous Treatment of Short and Long Scale Interfaces, Ph.D. thesis, FACULTAD DE INGENIERIA Y CIENCIAS HIDRICAS and INSTITUTO DE DESARROLLO TECNOLOGICO PARA LA INDUSTRIA QUIMICA, 2013.

[34] B. van Leer, Towards the ultimate conservative difference scheme, v. a second order sequel to godunov's method, Journal of Computational Physics 32 (1979) $101-136$.

[35] E. Goncalves, J. Decaix, Wall model and mesh influence study for partial cavities, European Journal of Mechanics, B/Fluids 31 (2012) 12-29.

[36] I. Abbot, A. von Doenhoff, E. Albert, Summary of airfoil data, Technical Report 824, NACA Report, 1945.

[37] F. Avellan, P. Henry, I. Ryhming, A new high speed cavitation tunnel, in: Proceedings of International Symposium on Cavitation Research Facilities and Techniques, ASME Winter Annual Meeting, volume 57, 1987, pp. 49-60.

[38] J. Jeong, F. Hussain, On the identification of a vortex, Journal of Fluid Mechanics 285 (1995) 69-94. doi:10.1017/S0022112095000462.

[39] P. Chakraborty, S. Balachandar, R. Adrian, On the relationships between local vortex identification schemes, Journal of Fluid Mechanics 535 (2005) 189-214.

[40] M. Lesieur, O. Métais, P. Comte, Large-Eddy Simulations of Turbulence, Cambridge University Press, 2005. Cambridge Books Online. 


\section{Appendix: simulation and solution convergence assessment}

The mesh convergence has been assessed by monitoring the time history of the drag coefficient (see figure 20)and of the mean streamwise velocity component located in $x / c=1.5, y / c=0$ and $z / c=0.1$ (see figure 21) over the averaging period of $T=0.1 \mathrm{~s}$ for the reference mesh. The drag coefficient is rather constant for the non-cavitating case over the averaging period with a value of $C_{d}=0.063$ and converge to a constant value around $C_{d}=0.116$ for the cavitating case. Regarding the velocity probe, the two configurations show a constant value of $\overline{u^{*}}=0.95$ for the non-cavitating case and $\overline{u^{*}}=1.025$ for the cavitating case. On a refined mesh with 6 million of nodes, the drag coefficient converge respectively to $C_{d}=0.063$ for the non-cavitating case and to $C_{d}=0.122$ for the cavitating case.

The vortex and cavitating regions are compared between the reference and the refined meshes on figure 22 by superposing on one hand an iso-surface of the Q-criterion and on another hand an iso-surface of the time-averaged liquid volume fraction $\alpha_{\text {Lmean }}$. For the two meshes, the iso-surfaces superimpose.

Finally, figure 23 displays the position of the TLV provided by the computations on the refined mesh. Without cavitation, the pressure minimum gives the same results than the Q-criterion and the maximum of the axial vorticity. Therefore, the small difference observed on the reference mesh (see figure 15) is cancelled on the refined mesh. This conclusion is not supported for the cavitating case since the minimum of the liquid volume fraction still provides a pitchwise and spanwise position of the TLV core lower than the Q-criterion. Nevertheless, the mesh refinement tends to bring closer the pressure minimum and the minimum of the liquid volume fraction. 
Table 1: Dimensionless pitchwise position $y / c$ of the vortex core at the experimental measurement sections.

\begin{tabular}{|l|c|c|c|}
\hline$x / c$ & Experiment & Non-cavitating RANS computation & Absolute error $\delta(\%)$ \\
\hline 0.1 & 0.141 & 0.132 & 6.4 \\
0.12 & 0.183 & 0.179 & 2.2 \\
0.15 & 0.303 & 0.217 & 28.4 \\
\hline
\end{tabular}


Table 2: Dimensionless spanwise position $z / c$ of the vortex core at the experimental measurement sections.

\begin{tabular}{|l|c|c|c|}
\hline$x / c$ & Experiment & Non-cavitating RANS computation & Absolute error $\delta(\%)$ \\
\hline 0.1 & 0.120 & 0.107 & 11.0 \\
0.12 & 0.132 & 0.122 & 7.6 \\
0.15 & 0.16 & 0.149 & 6.9 \\
\hline
\end{tabular}



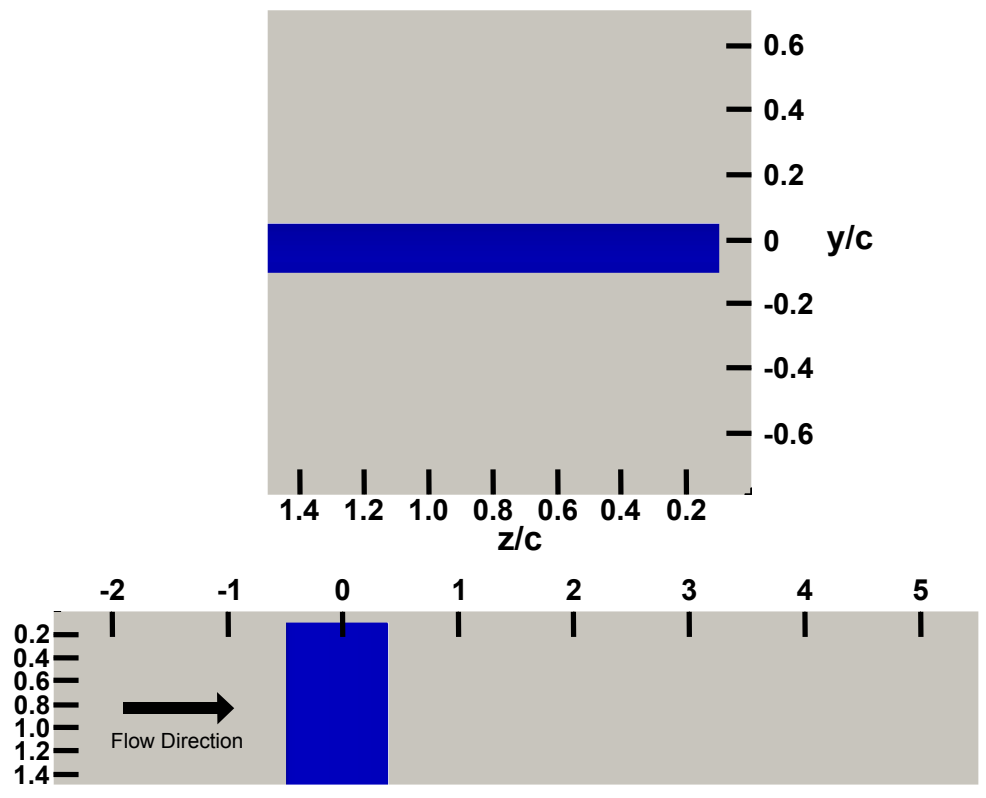

Figure 1: Computational domain. Downstream view (top), top view (bottom). 


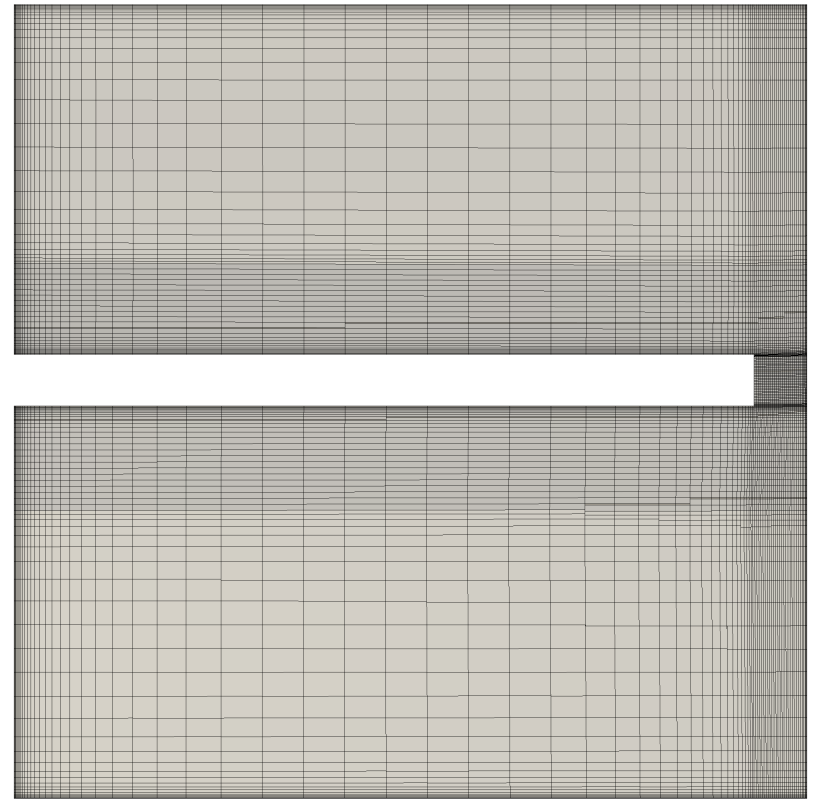

Figure 2: View of the mesh in a cross section located at mid-chord. 


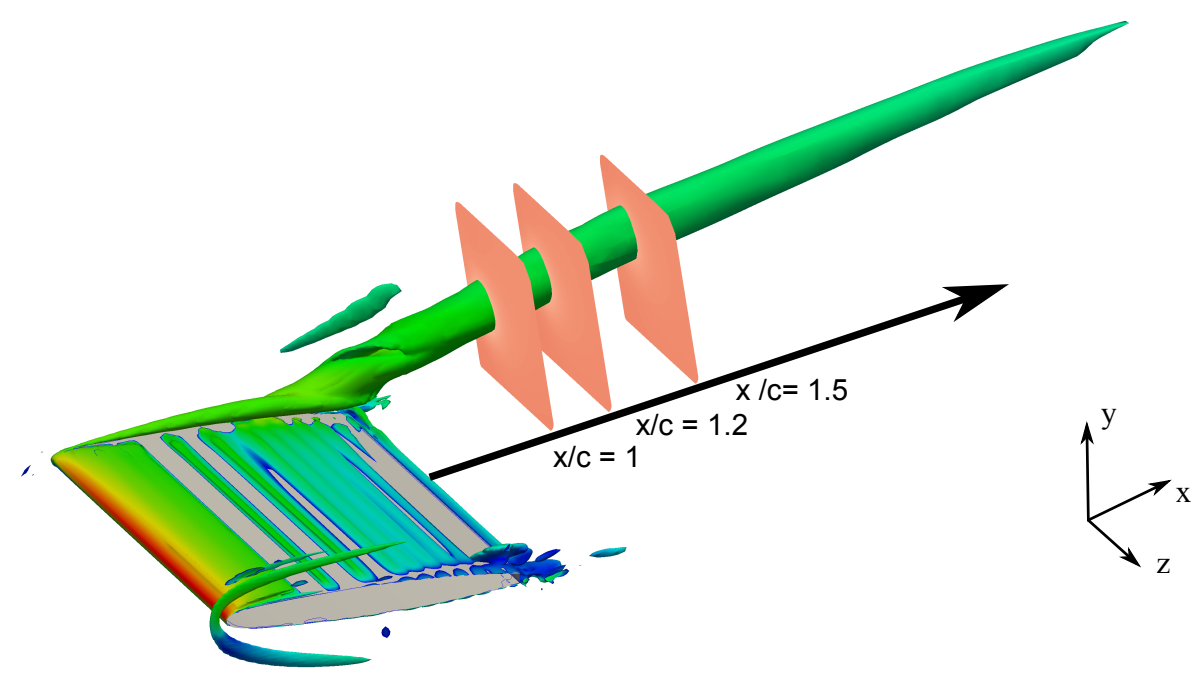

Figure 3: Positions of the measurements section illustrated using an iso-surface of the Q-criterion (Non-cavitating URANS computation). 

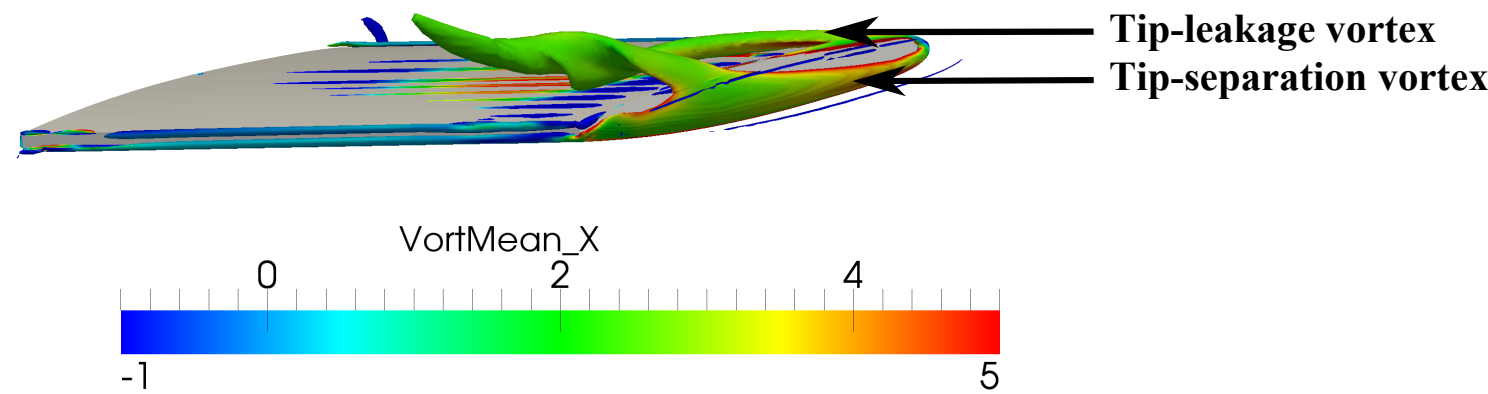

Figure 4: Iso-surface of the dimensionless Q-criterion $\left(Q^{*}=1.5\right)$ computed with the time-averaged velocity and coloured by the dimensionless time-averaged axial vorticity. Non-cavitating URANS computation. 

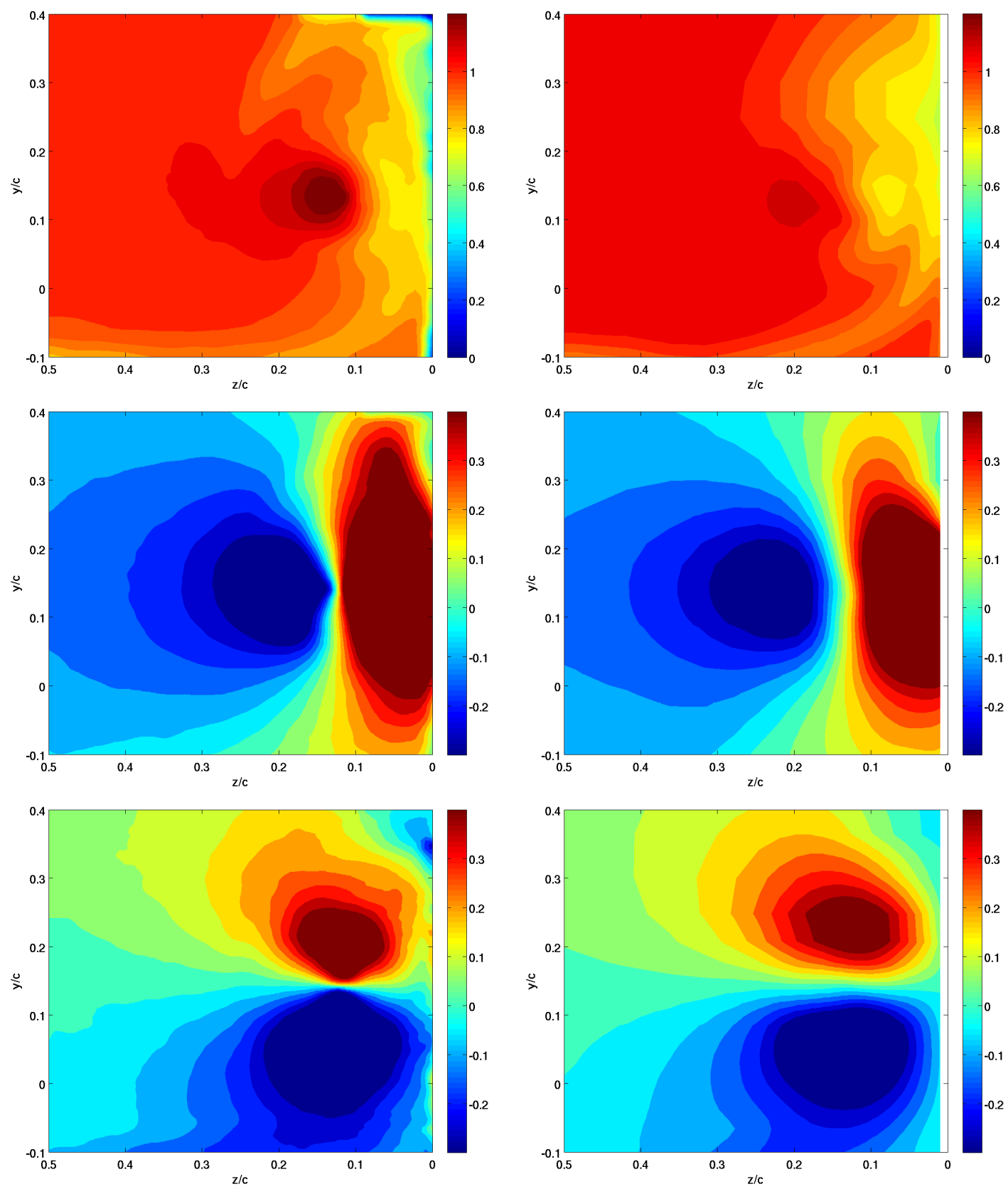

Figure 5: Dimensionless time-averaged velocity component ( $\overline{u^{*}}$ top, $\overline{v^{*}}$ middle and $\overline{w^{*}}$ bottom) at the cross plane located at $x / c=1$. Experiment (left) and non-cavitating URANS computation (right). 

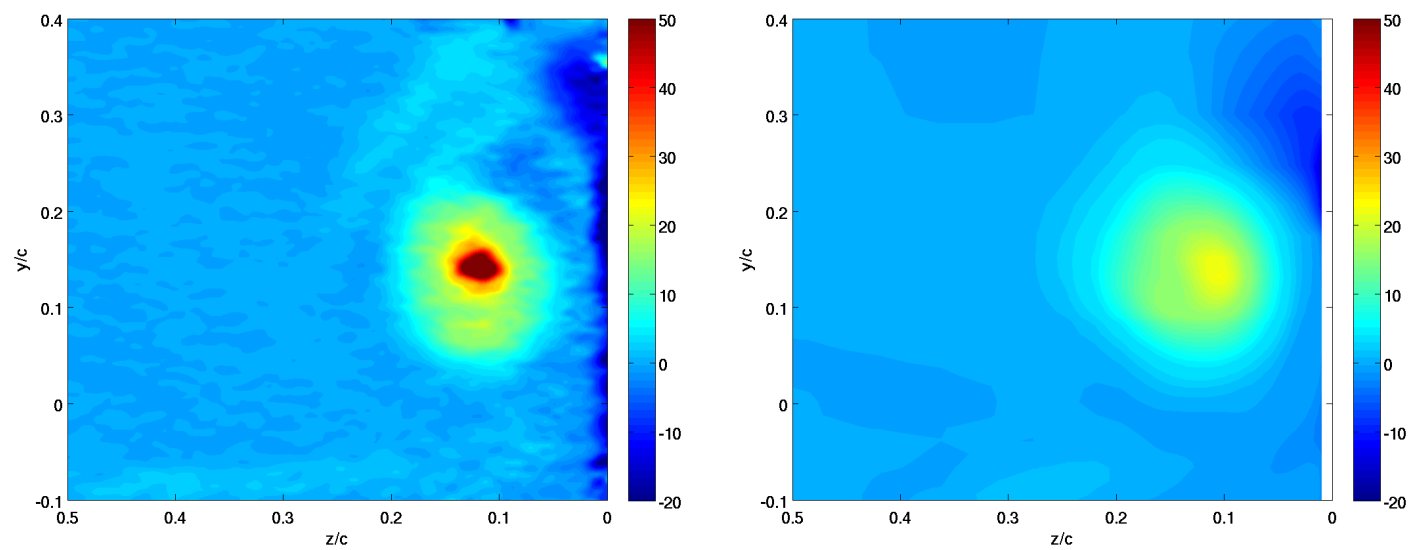

Figure 6: Dimensionless time-averaged axial vorticity $\overline{\omega_{x}^{*}}$ at the cross plane located at $x / c=1$. Experiment (left) and non-cavitating URANS computation (right). 


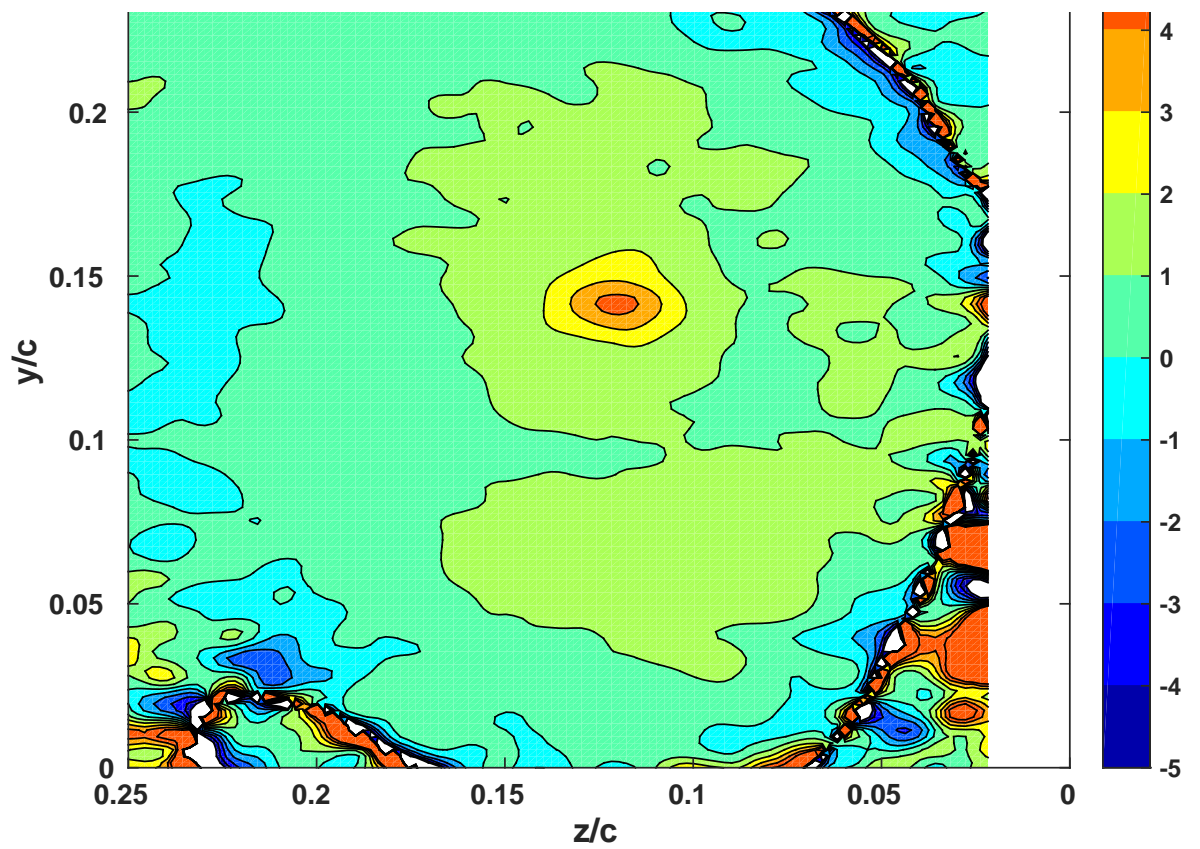

Figure 7: Ratio between the experimental and numerical dimensionless time-averaged axial vorticity $\left(\overline{\omega_{x_{\text {expe }}}^{*}} / \overline{\omega_{x_{R A N S}}^{*}}\right)$ at the cross plane located at $x / c=1$. 

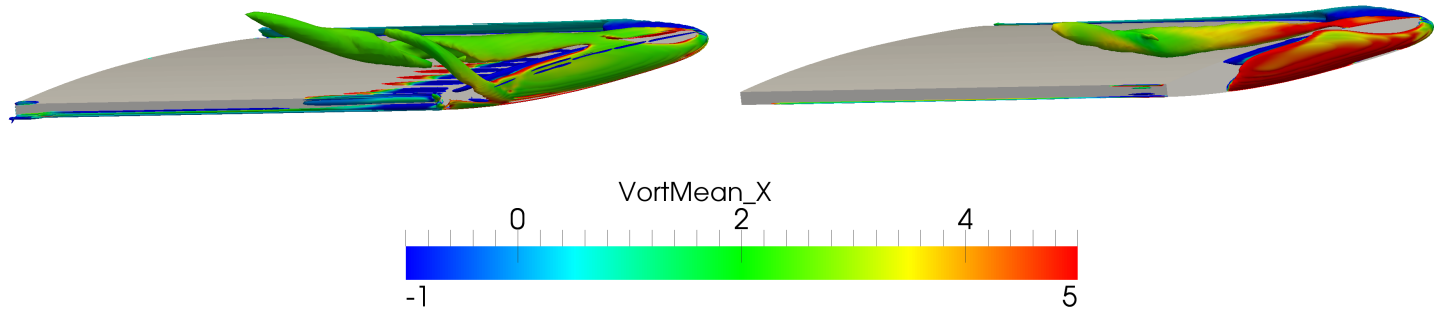

Figure 8: Iso-surface of the dimensionless Q-criterion $Q^{*}=1.5$ (left) and iso-surface of the timeaveraged liquid volume fraction $\alpha_{L \text { mean }}=0.9$ (right), both coloured by the the dimensionless time-averaged axial vorticity. Cavitating URANS computation. 

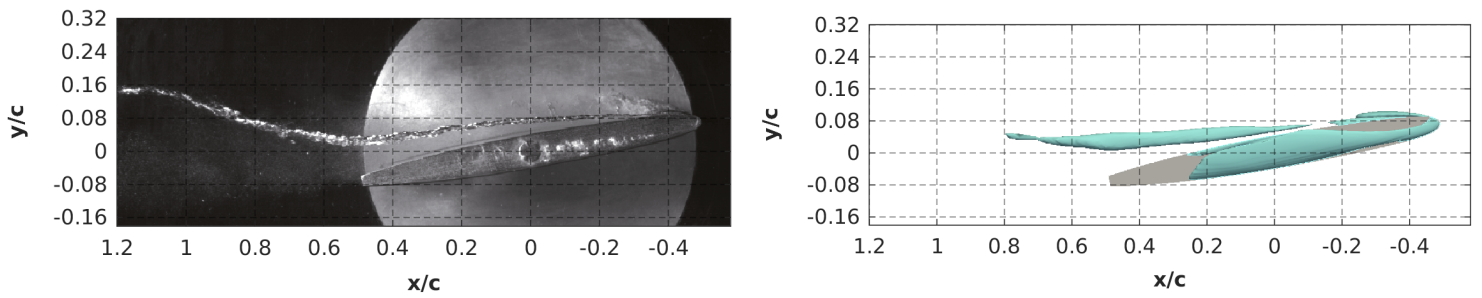

Figure 9: Instantaneous experimental picture (left) and iso-surface of the time-averaged liquid volume fraction $\alpha_{L \text { mean }}=0.5$ (right) provided by the cavitating URANS computation. 

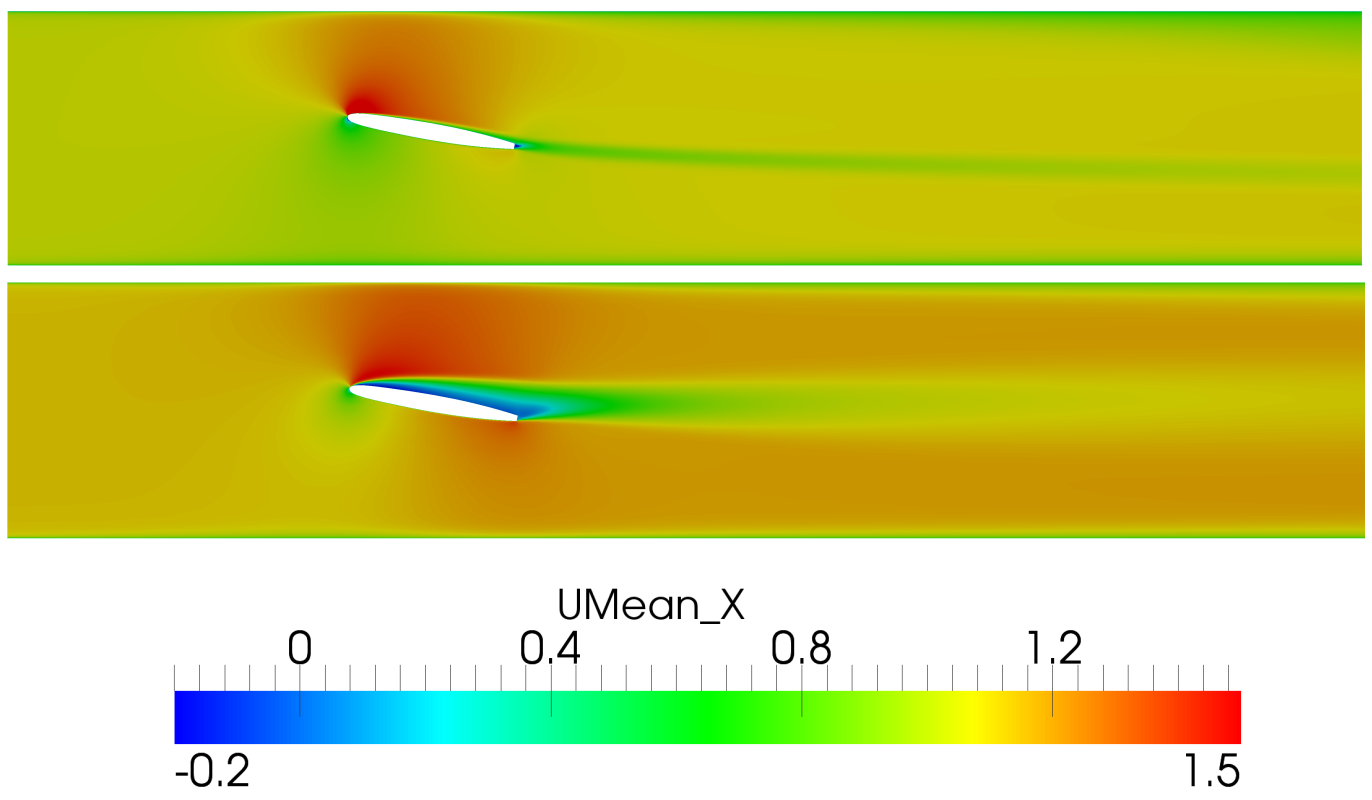

Figure 10: Dimensionless time-averaged streamwise velocity component in the mid plane. Noncavitating RANS computation (top) and cavitating URANS computation (bottom). 

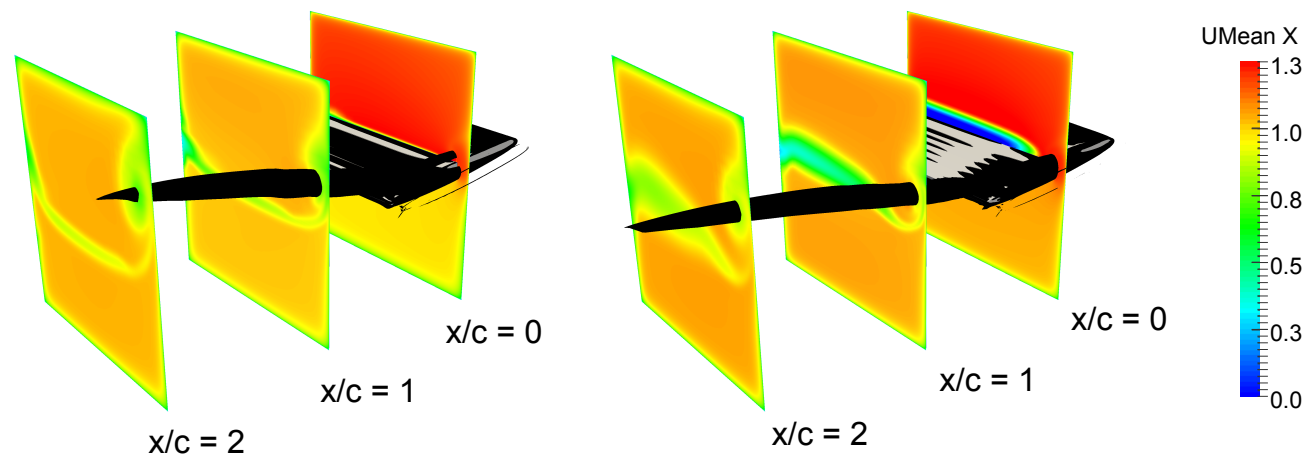

Figure 11: Dimensionless time-averaged streamwise velocity component in three cross planes with an iso-surface of the dimensionless Q-criterion $\left(Q^{*}=0.2\right)$ in black. Non-cavitating (left) and cavitating URANS computations (right). 

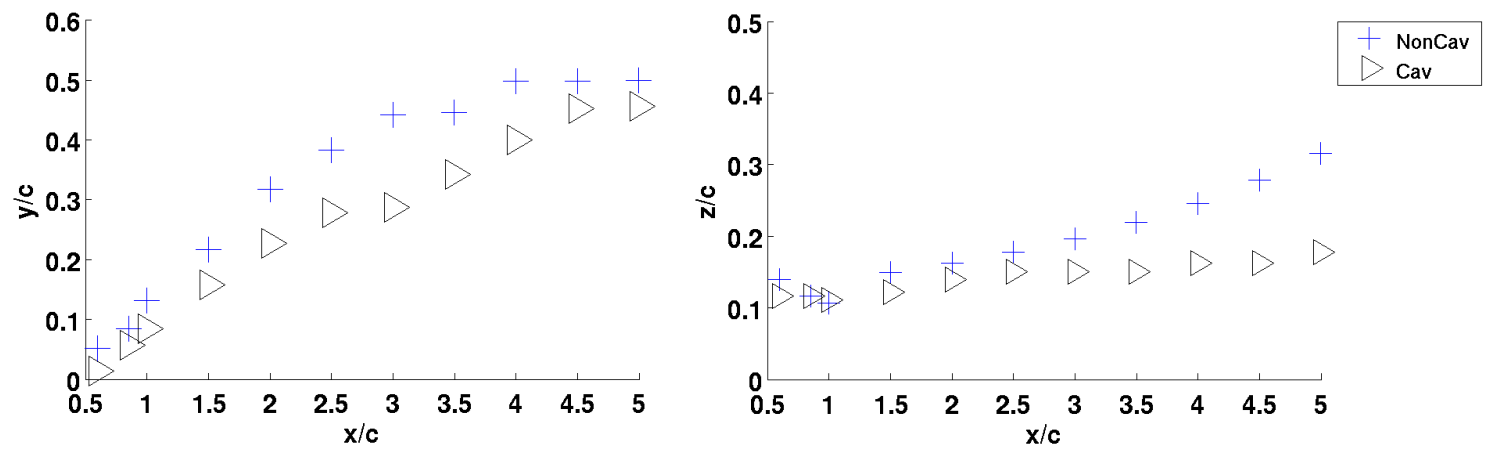

Figure 12: Pitchwise (left) and spanwise (right) position of the vortex core downstream the trailing edge using the $Q$ criterion. 

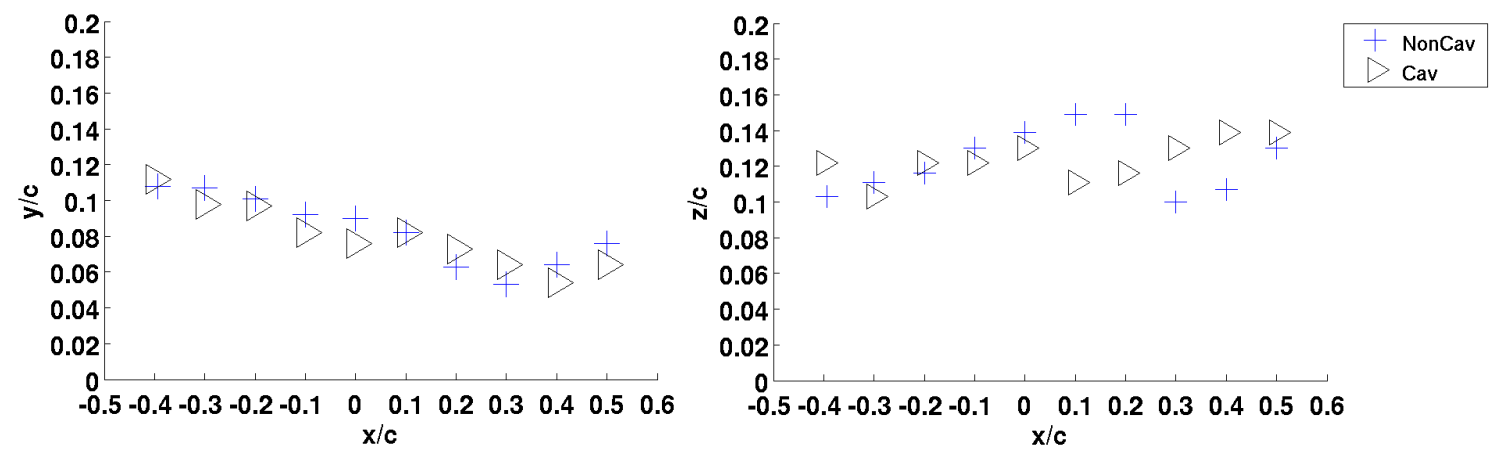

Figure 13: Pitchwise (left) and spanwise (right) position of the tip-leakage vortex core above the blade using the $Q$ criterion. 


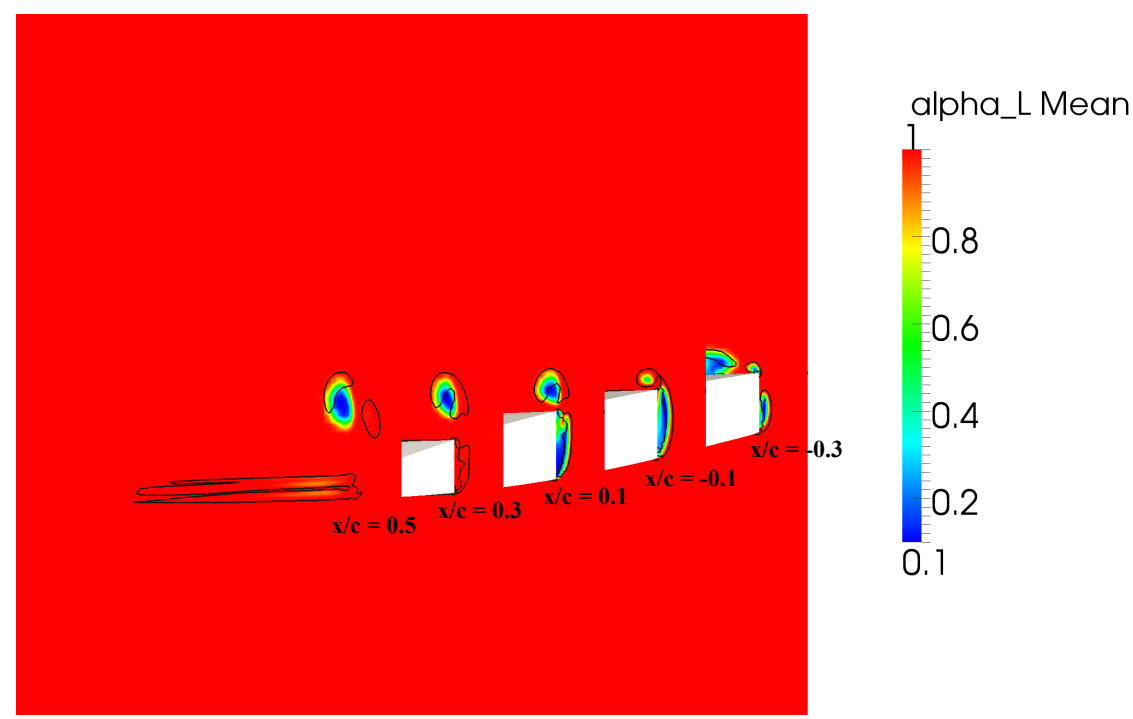

Figure 14: Contours of the time-averaged liquid volume fraction with an iso-contour of the dimensionless Q-criterion $\left(Q^{*}=1.5\right)$. Cavitating RANS computation. 

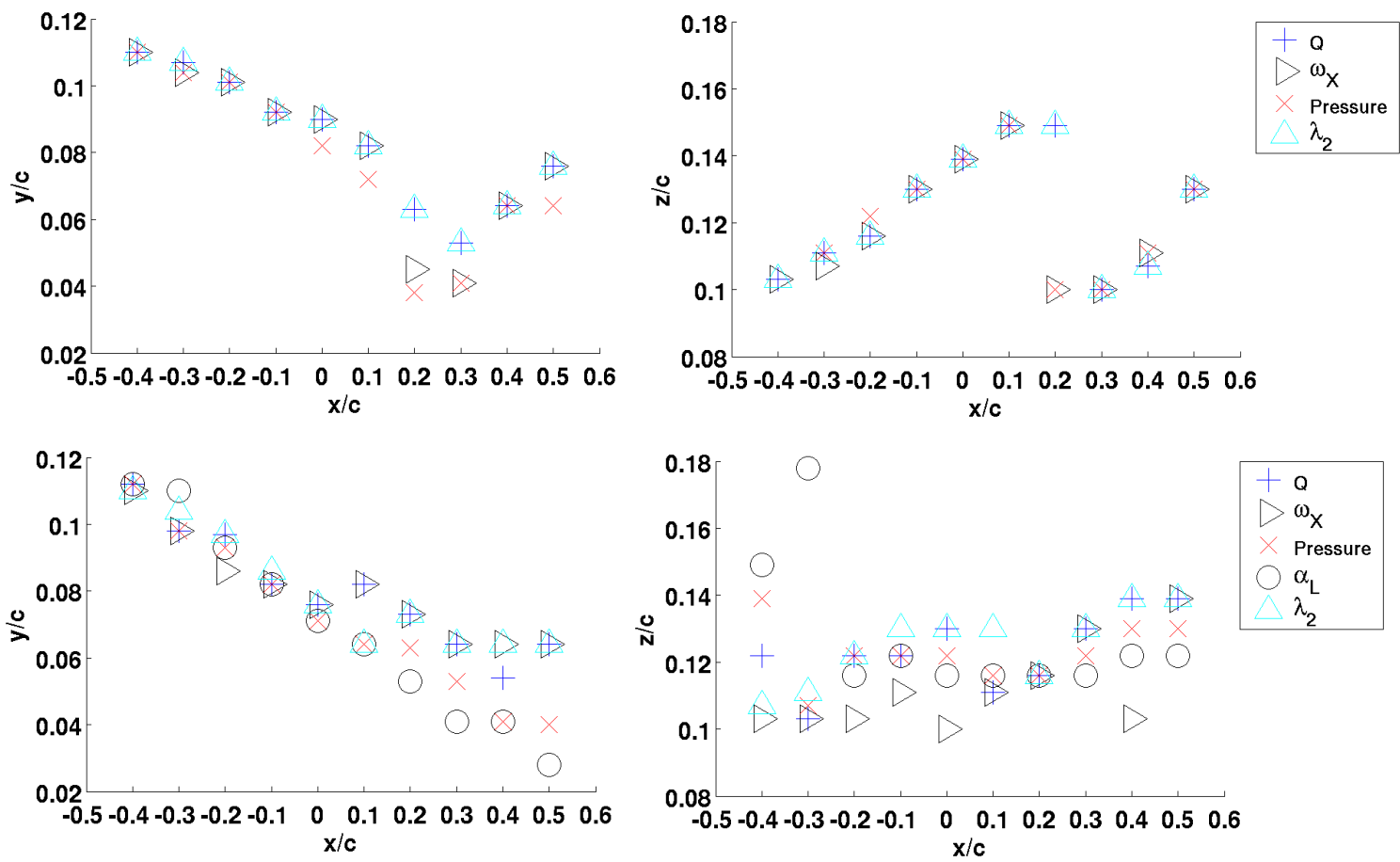

Figure 15: Pitchwise (left) and spanwise (right) position of the tip-leakage vortex core identified using various criteria. Non-cavitating (top) and cavitating (bottom) URANS computations. 

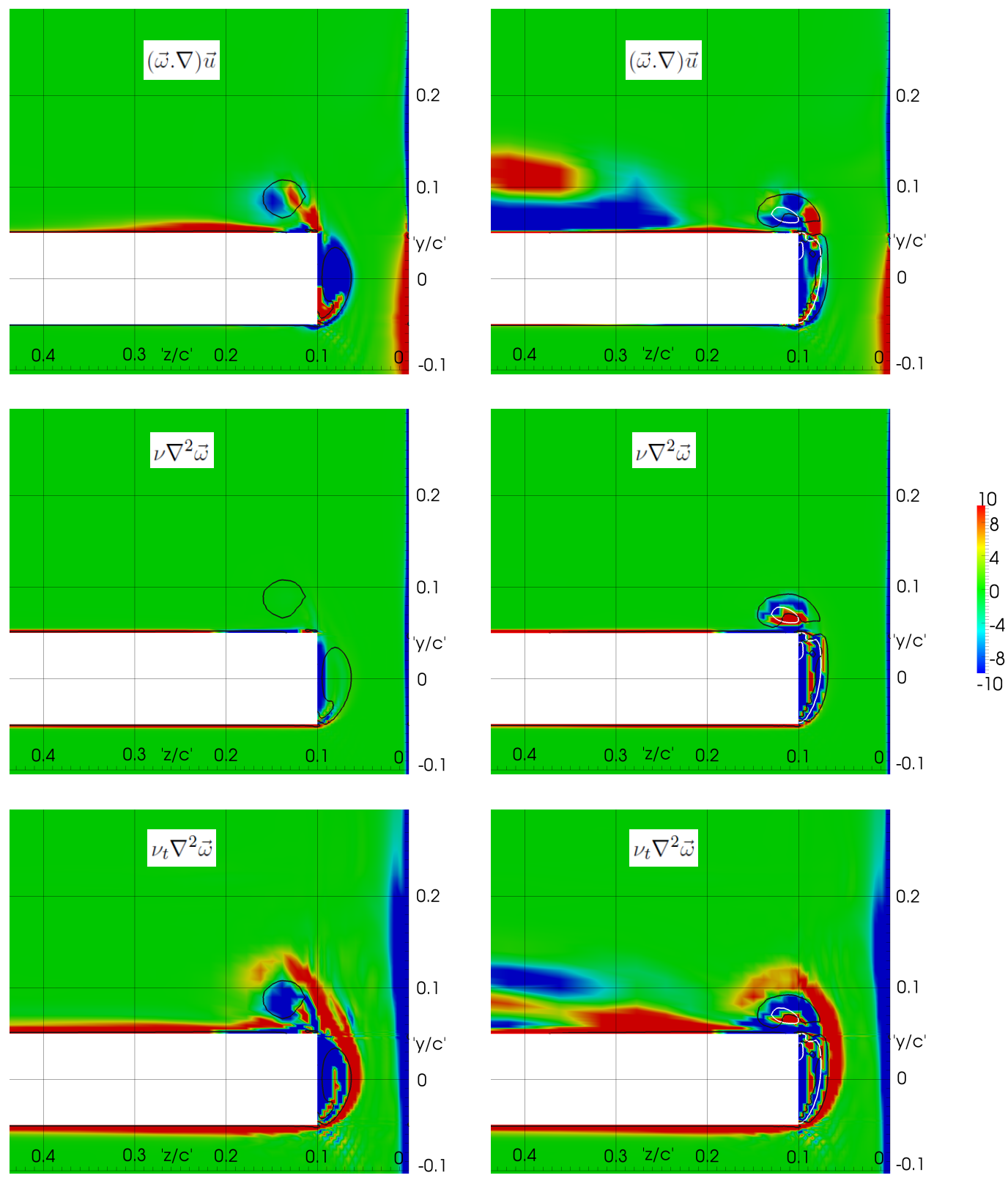

Figure 16: Dimensionless streamwise component of the vortex stretching, viscous and turbulent terms of the vorticity equation in a $y z$-plane at $x / c=0$. Black line refers to the iso-value of the Q-criterion $\left(Q^{*}=1.5\right)$. White line refers to the iso-value of the liquid volume fraction $\left(\alpha_{L}=0.5\right)$. Non-cavitating (left) and cavitating (right) URANS computations. 

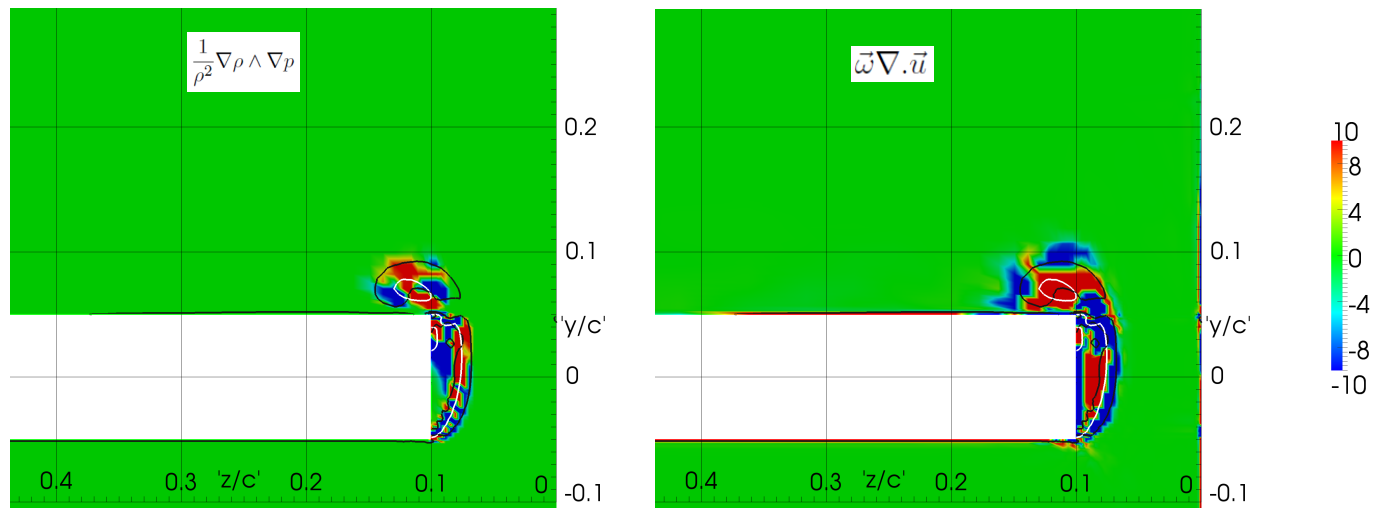

Figure 17: Dimensionless streamwise component of the baroclinic torque and the compressible term of the vorticity equation in a $y z$-plane at $x / c=0$. Black line refers to the iso-value of the Qcriterion $\left(Q^{*}=1.5\right)$. White line refers to the iso-value of the time-averaged liquid volume fraction $\left(\alpha_{\text {Lmean }}=0.5\right)$. Cavitating URANS computations. 

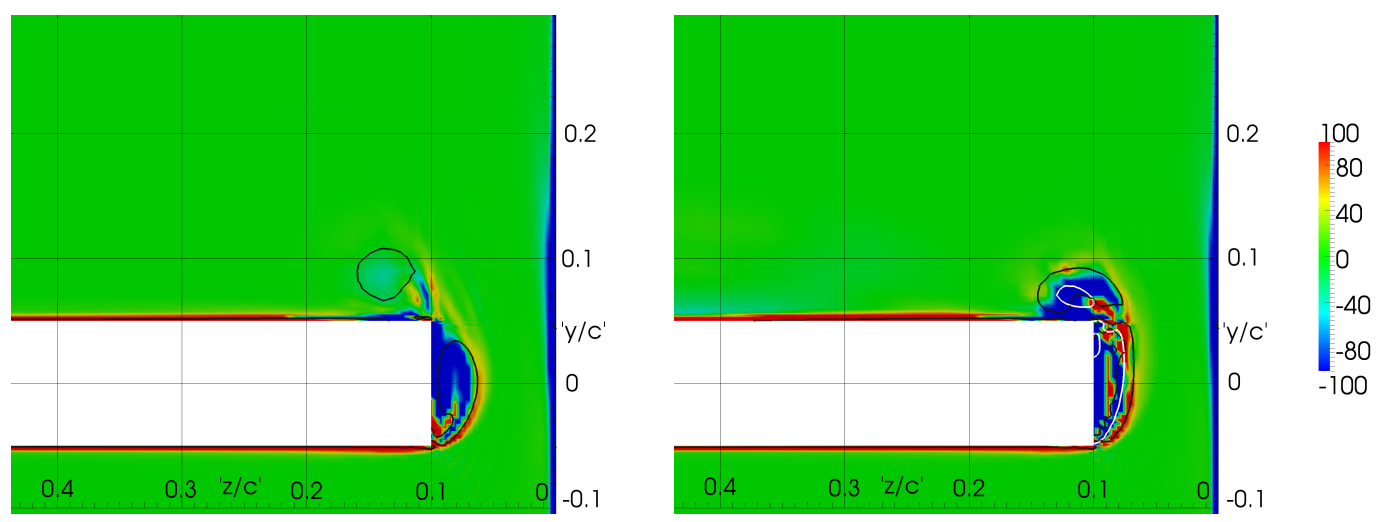

Figure 18: Dimensionless streamwise component of the right-hand side term of the vorticity equation in a $y z$-plane at $x / c=0$. Black line refers to the iso-value of the $\mathrm{Q}$-criterion $\left(Q^{*}=1.5\right)$. White line refers to the iso-value of the time-averaged liquid volume fraction $\left(\alpha_{\text {Lmean }}=0.5\right)$. Non-cavitating (left) and cavitating (right) URANS computations. 

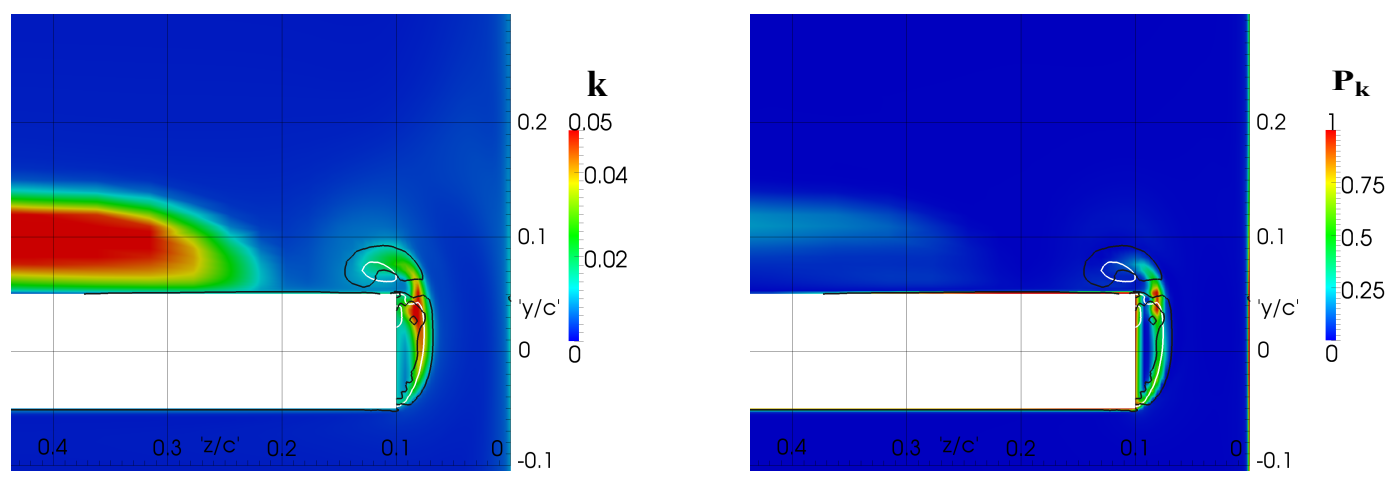

Figure 19: Dimensionless time-averaged turbulent kinetic energy and dimensionless turbulent kinetic production term in a $y z$-plane at $x / c=0$. Black line refers to the iso-value of the Qcriterion $\left(Q^{*}=1.5\right)$. White line refers to the iso-value of the time-averaged liquid volume fraction $\left(\alpha_{\text {Lmean }}=0.5\right)$. Cavitating URANS computations. 


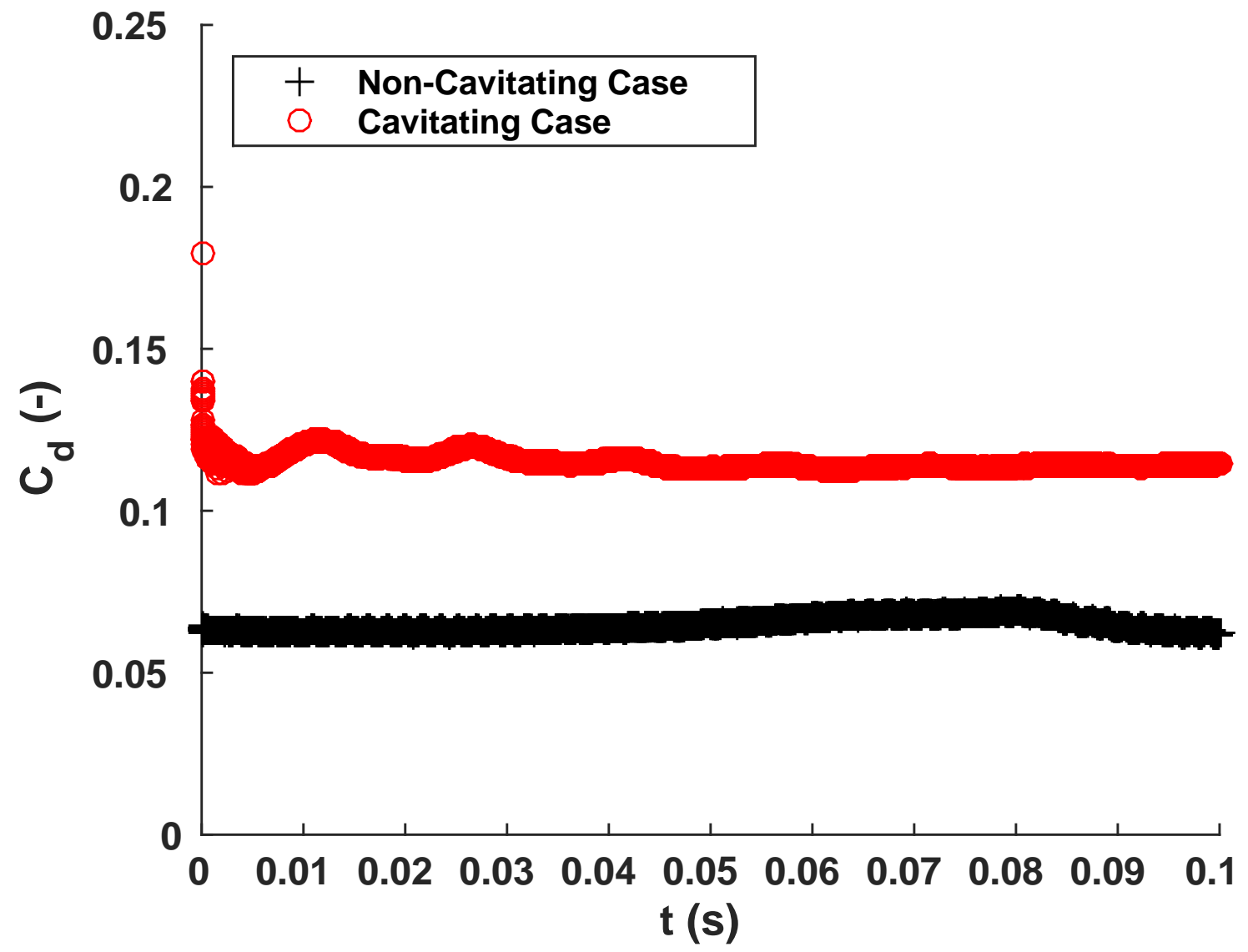

Figure 20: Time history of the drag coefficient. URANS computations on the reference mesh. 


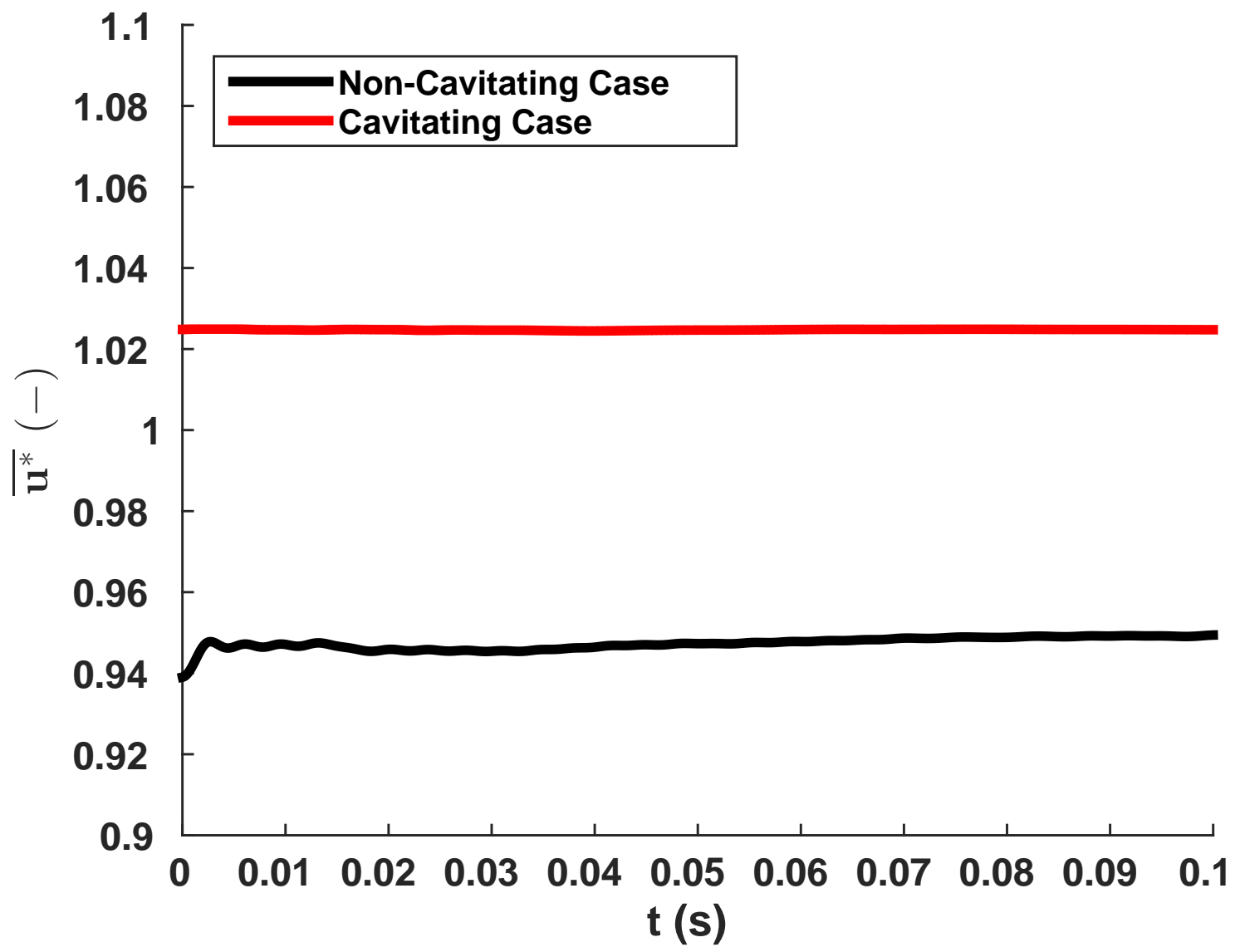

Figure 21: Time history of the mean velocity component $\overline{u^{*}}$ located in $x / c=1.5, y / c=0$ and $z / c=0.1$. URANS computations on the reference mesh. 

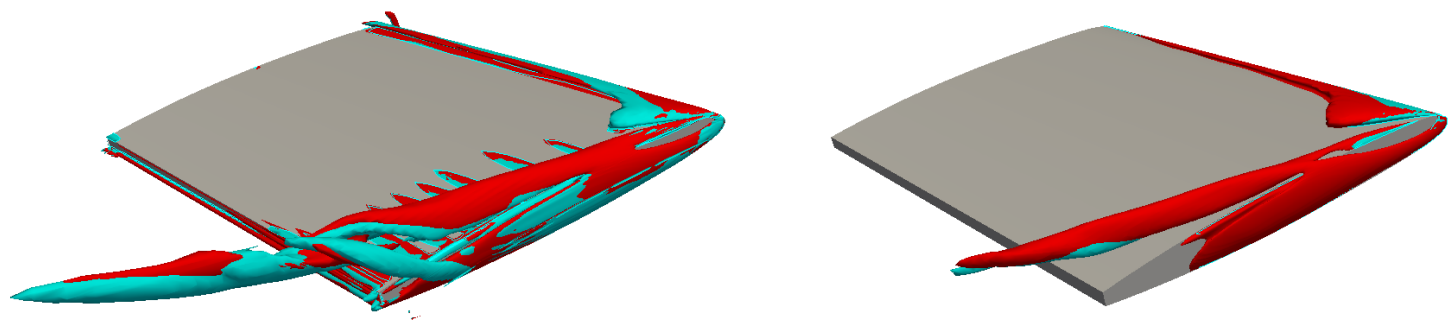

Figure 22: Comparison of the iso-surface of the dimensionless Q-criterion $Q^{*}=1.5$ (left) and of the iso-surface of the time-averaged liquid volume fraction $\alpha_{\text {Lmean }}=0.5$ (right) between the reference mesh (light blue) and the refined mesh (red). Cavitating URANS computations. 

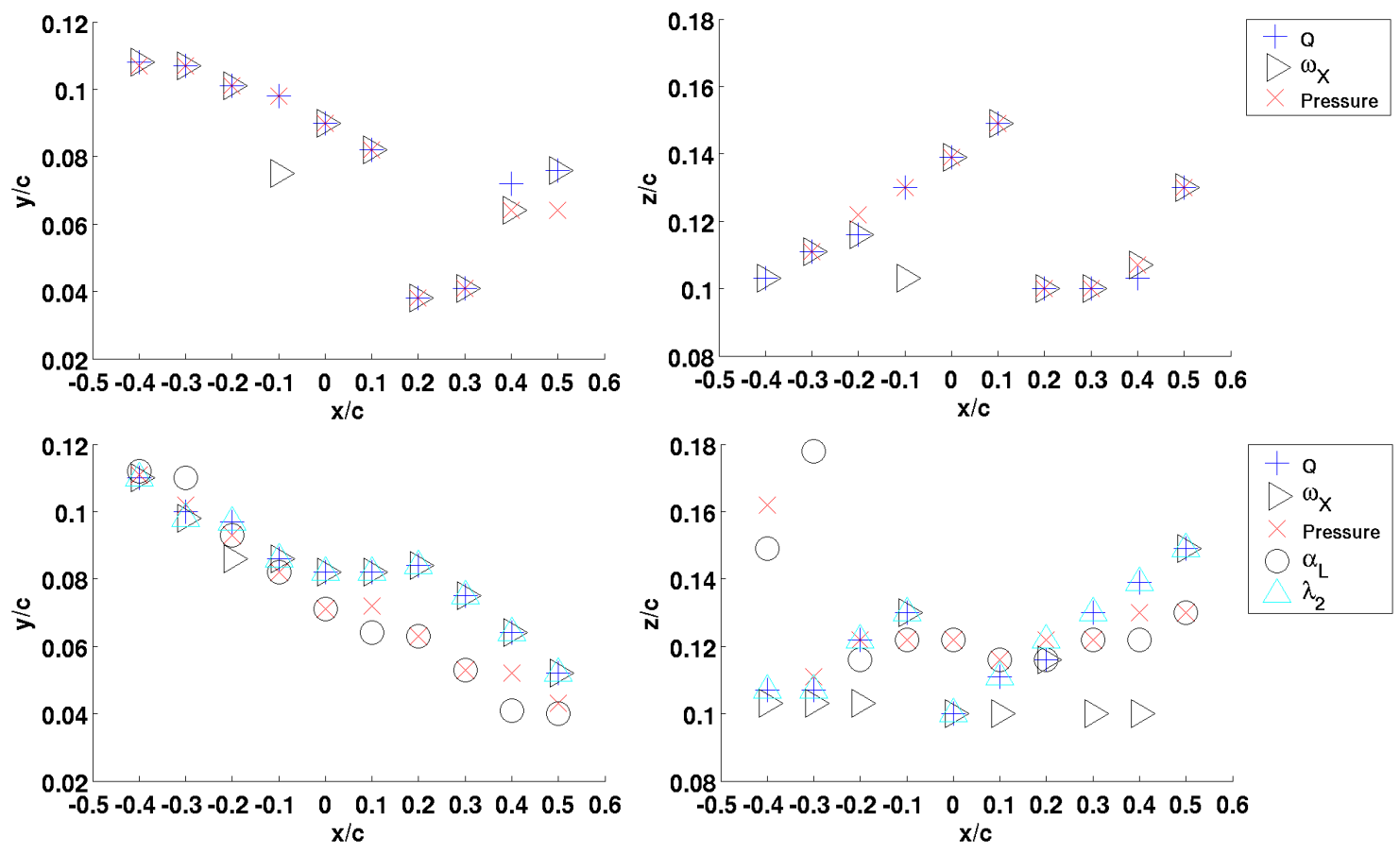

Figure 23: Pitchwise (left) and spanwise (right) position of the tip-leakage vortex core identified using various criteria. Non-cavitating (top) and cavitating (bottom) URANS computations on the refined mesh. 